\title{
Changes in volatile compounds of Ayvalık (Edremit) and Uslu olive oils depending on conditions and time of storage
}

\author{
Pelin Günç Ergönül ${ }^{1}$, Alev Yüksel Aydar ${ }^{1}$, \\ Tuba Göldelí ${ }^{1}$, Annalisa Mentana ${ }^{2}$, Maurizio Quinto
}

\author{
1 - Manisa Celal Bayar University, Manisa, Turkey \\ 2 - Foggia University, Foggia, Italy
}

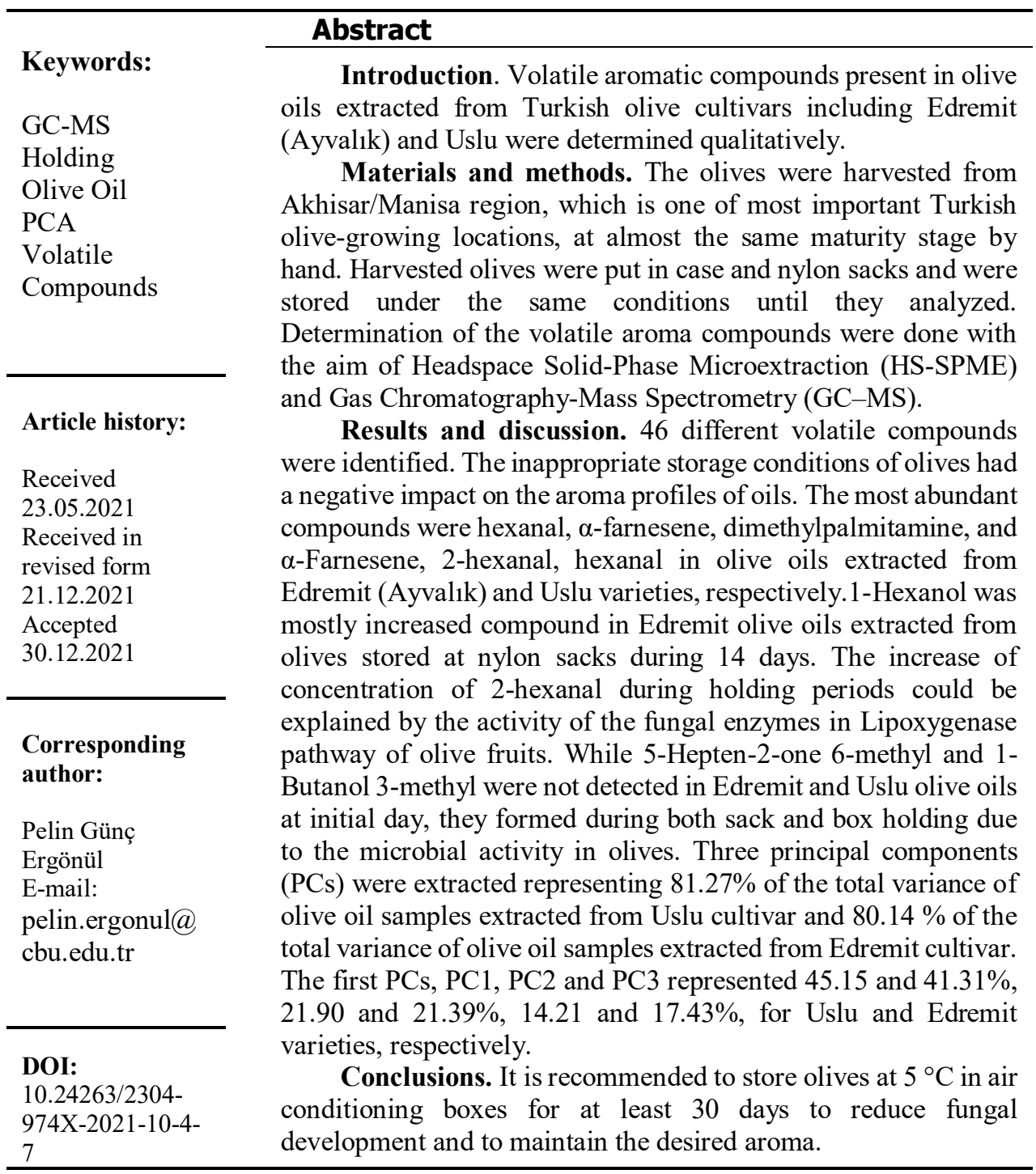




\section{Introduction}

Olive fruit (Olea europaea) is one of most important products in Mediterranean countries and olive cultivation and processing has been carried out since the beginning of human civilization (Ozdemir et al. 2018). Olive and olive oil have superior nutritional properties due to high content of volatile and phenolic compounds, so their regular consumprion could help in prevention of such diseases such as cardiovascular diseases, cancer and osteoporosis. Production of a high quality virgin olive oil requires the storing the olive fruit in proper holding conditions (Inarejos-García et al. 2009). In addition to the extraction process and storage, cultivar and cultivar applications, geographical origin, climatic conditions, degree of fruit ripening also affect the content and composition of volatiles compounds in olive oils (Issaoui et al. 2010; Kesen et al. 2013). Several researchers have determined that the olive storage types and times affect the virgin olive oil quality as much as technological operations (Bouaziz et al. 2008; Inarejos-García et al. 2009; Pereira et al. 2002; Rizzo et al. 2011).

The presence of volatile and phenolic compounds directly influence and determine the quality of virgin olive oil. Volatile aromatic compounds are one of the most important factors for olive oil's quality and affect sensorial perception (Bayrak \& Hu 2013). They are formed by the oxidation of oils with certain enzymes such as lipoxygenase (LOX pathway) (Cavalli et al. 2004). Olive oils possess more than 180 different aromas, and the majority of the volatile substances are presented by esters, aldehydes, hydrocarbons, ketones, and furans (Kesen et al. 2013). Servili et al (2003) studied the time of revealing of olive pastes to air during malaxation and found a positive correlation between air contact and the content of volatile compounds in olive oil including hexanal, 1-butanol, (Z)-3-hexen-1-ol, 1-penten-3$\mathrm{ol}$, and 2-methyl-1-butanol. Inappropriate olive fruit storage generally induce the activity of microorganisms that are responsible for unpleasant odours and formation of volatile compounds (Koprivnjak \& Conte 2002).

Principal component analysis (PCA) is an statistical method that can be used to detemine the content of triglycerides, sterols, phenolic compounds and volatile compounds to distinguish oils from different cultivars (Boskou 2007; de Fernandez et al. 2014; Yang et al. 2017). The aim of this study was to examine the effects of different holding times and types on volatile aroma compounds of oil samples obtained from different olive cultivars named "Edremit and Uslu" collected from Aegean province, Akhisar Region of Turkey by Gas Chromatography- Mass Spectroscopy (GC-MS) headspace technique. There is no detailed information that compares the volatile profiles of oils extracted from olives holded in unsuitable conditions that is already applied by some olive producers. For this reason, this study carried out is of importance.

\section{Materials and methods}

\section{Materials}

Olive fruits (Olea europaea L.) from the Edremit and Uslu varieties grown in the Akhisar area were harvested in the 2012/2013 crop season. Maturity index of olives, which was calculated according to the method of the International Olive Oil Council (IOOC, 2011), was $3.75 \pm 0.35$ and $4.50 \pm 0.71$ for Edremit and Uslu cultivars, respectively. According to this method, 100 fruits were randomly taken to assess their level of maturity by a subjective evaluation of the color of the olive skin and flesh. The olives were distributed into eight 
groups according to the following characteristics: bright-green skin (group $\mathrm{N}$ 0), greenishyellowish skin (group N 1), green skin with reddish spots (group N 2), reddish-brown skin (group N 3), black skin with white flesh (group N 4), black skin with $<50 \%$ purple flesh (group N 5), black skin with $>50 \%$ and $<100 \%$ purple flesh (group $\mathrm{N} 6$ ), and black skin with $100 \%$ purple flesh (group N 7). Maturation indexes ranged from zero (intense green skin) to seven (black skin and 100\% purple flesh). The maturity index was calculated by $\sum$ $\left(\mathrm{N}_{\mathrm{i}} \mathrm{n}_{\mathrm{i}}\right) / 100$, where $\mathrm{N}$ is the group number and $\mathrm{n}$ is the olive amount in that group (Aydar et al. 2017). Air temperature and weather conditions during olives holded periods were shown in Table 1 (Turkish State Meteorological Service, 2012-2013). Olives were put into nylon sacks $(60 \times 90 \mathrm{~cm})$ and plastic boxes $(53 \times 37 \times 31 \mathrm{~cm})$ and were hold inside them for $0,7,14$ and 21 days. Olive oil samples symbolized as E: Edremit variety, U: Uslu variety, K: Holded in plastic boxes, and C: Holded in nylon sacks.

Air temperature and weather conditions during olives holded periods

Table 1 (Turkish State Meteorological Service, 2012-2013)

\begin{tabular}{|l|l|l|}
\hline MANISA/AKHISAR & $\begin{array}{l}\text { Air } \\
\text { temperature } \text { ( }^{\circ} \text { C) }\end{array}$ & Weather \\
\hline $\begin{array}{l}\mathbf{2 1 . 1 2 . 2 0 1 2} \\
\text { (harvest-first extraction) }\end{array}$ & 7 & Snowy \\
\hline 1th day & 8 & Sunny \\
\hline 2th day & 8 & Sunny \\
\hline 3th day & 7 & Sunny \\
\hline 4th day & 12 & Sunny \\
\hline 5th day & 16 & Sunny \\
\hline 6th day & 15 & Partly cloudy \\
\hline $\begin{array}{l}\mathbf{2 8 . 1 2 . 2 0 1 2} \\
\text { (7th day-second extraction) }\end{array}$ & 14 & Stormy \\
\hline 8th day & 13 & Stormy \\
\hline 9th day & 12 & Stormy \\
\hline 10th day & 13 & Stormy \\
\hline 11th day & 13 & Some clouds \\
\hline 12th day & 13 & Some clouds \\
\hline 13th day & 13 & Some clouds \\
\hline $\begin{array}{l}\mathbf{0 4 . 0 1 . 2 0 1 3} \\
\text { (14th day-third extraction) }\end{array}$ & 10 & Foggy \\
\hline 15th day & 11 & Some clouds \\
\hline 16th day & 10 & Some clouds \\
\hline 17th day & 6 & Partly cloudy \\
\hline 18th day & 6 & Partly cloudy \\
\hline 19th day & 3 & Partly cloudy \\
\hline 20th day & 5 & Partly cloudy \\
\hline $\begin{array}{l}\text { 11.01.2013 } \\
\text { (21th day-fourth extraction) }\end{array}$ & 8 & Stormy \\
\hline & & \\
\hline
\end{tabular}




\section{Methods}

\section{Oil extraction}

A laboratory scale Abencor extraction system consisted of a small-quantity mill (MC2 Ingenieria Sistemas, Seville, Spain) equipped with a mixer (Kitchen Aid Mixer 4lt Model 5KSM45 220-240VN 50-60 HZ 250W, USA), a basket centrifuge (Marelli Motore Asinciono Trifase Tipo NR90S2) and a metal crusher was used to extract oil from olive fruits. The malaxation process was performed at $35 \pm 1{ }^{\circ} \mathrm{C}$ for $60 \mathrm{~min}$ for Edremit variety and at $35 \pm 1{ }^{\circ} \mathrm{C}$ for $90 \mathrm{~min}$ for Uslu variety, and the oil separation was carried out by decanter. Extracted olive oils were filtered and then kept at $4^{\circ} \mathrm{C}$ in amber glass bottles until analysis.

\section{Volatile compound analysis}

Sample Preparation. An $8 \mathrm{~mL}$ of oil was put in a $20 \mathrm{~mL}$ glass headspace sample vial and to attain a final $3 \mathrm{ppm}$ concentration, $27 \mu \mathrm{L}$ and $24 \mu \mathrm{L}$ of internal standard solutions (butyl acetate and 1-nonanol, respectively) were added to each vial. The mixture was shaken carefully and allowed to equilibrate for 1 hour in the dark at ambient temperature before the analysis.

HS-SPME procedures. The SPME coated by polydimethylsiloxane (PDMS) fiber at $100 \mu \mathrm{m}$ thickness and 23 gauge was used in this study. It was purchased from Supelco and thermal conditions provided according to the manufacturer's recommendations before first use. The samples of oils were heated to $40{ }^{\circ} \mathrm{C}$ for $10 \mathrm{~min}$ before revealing the SPME fiber to the headspace of the sample. Headspace sampling/extraction took $30 \mathrm{~min}$ with continuous stirring $(250 \mathrm{rpm})$. The samples were analyzed in duplicate and as a blank sample (empty glass vial) was used before and after each analysis.

GC-MS analysis. The analytical system was constituted from A Gerstel MPS autosampler (Gerstel, Baltimore, MD, USA) installed to an Agilent $6890 \mathrm{~N}$ model Gas Chromatography (Little Falls, DE, USA) paired with an Agilent 5975 mass selective detector. The software was MSD ChemStation (Agilent). SPME injections were carried on a splitless mode using a SPME injection sleeve $\left(0.75 \mathrm{~mm}\right.$ I.D) at $250{ }^{\circ} \mathrm{C}$ for $350 \mathrm{sec}$ meanwhile thermal desorption of analyses was occurred in DB-Wax column $(60 \mathrm{~m} \times 0.25 \mathrm{mmI}$.D., $0.25 \mu \mathrm{m}$ film thickness) (J\&W Scientific, Folsom, CA, USA). Gas helium was used as a carrier with a total flow of $1.2 \mathrm{~mL} \cdot \mathrm{min}^{-1}$. The initial temperature $40{ }^{\circ} \mathrm{C}$ was kept for $1.0 \mathrm{~min}$, followed by an increased to $200{ }^{\circ} \mathrm{C}$ at a rate of $6^{\circ} \mathrm{C} \mathrm{min}^{-1}$ and kept at this temperature for $5 \mathrm{~min}$, then raised to $250{ }^{\circ} \mathrm{C}$ at a rate of $8{ }^{\circ} \mathrm{C} \mathrm{min}{ }^{-1}$. Lastly, the the temperature was retained $250{ }^{\circ} \mathrm{C}$ for $10 \mathrm{~min}$. The total cycle time was $48.92 \mathrm{~min}$. The MS detector was handled in scan mode (mass range $30-500$ ) and the transfer line to the MS system was retained at $250{ }^{\circ} \mathrm{C}$. The identification of the compounds was carried out by comparing (i) the Kovats indices (KI) based on a homologous series of even numbered n-alkanes $\left(\mathrm{C}_{8}-\mathrm{C}_{20}\right)$, with those of standard compounds and by comparison with the data of literature, and (ii) by MS data received from NIST library (NIST/EPA/NIH Mass Spectral Library with Search Program, data version NIST 05, software version $2.0 \mathrm{~d}$ ). 


\section{Statistical analysis}

XLSTAT (Addinsoft SARL, NY, USA) for Microsoft Excel (Microsoft, Redwood, WA) was used to perform ANOVA. In order to identify the variations of headspace components and analyze the composition data in different samples of oil, principal component analysis (PCA) was performed by SCAN software (Minitab Inc., State College, PA, USA). The software autoscaled the content values before the statistical analysis, i.e. each variable was subtracted by the mean value and the result was divided by its standard deviation.

\section{Results and discussion}

Principal volatile compounds are commonly found in great sensory quality virgin olive oil that are synthetized by biogenic pathways of the olive fruit such as fatty acid or amino acid metabolism and LOX pathway (Morales 2005). In spite of that, especially storing types and conditions, climatic conditions are very effective on producing of disagreeable volatiles compounds (Gomes da Silva et al. 2012). In olive fruits stored in batches, under high humidity conditions, the most abundant deuteromycetes such as several species of genus Aspergillus, together with ascomycetes, Penicillium notatum are occurred. These microorganisms have the capacity to oxidise free fatty acids and produce volatile compounds such as methyl ketones (Morales 2005).

In this study, 46 different volatile compounds were identified during different holding times of olive oils extracted from Edremit and Uslu varieties (Table 2, 3 and 4).

Volatile compounds isolated from Edremit (Ayvalık) and and Uslu oils

Table 2*

\begin{tabular}{|l|l|c|c|c|l|}
\hline Codes & \multicolumn{1}{|c|}{ Compounds } & $\begin{array}{c}\text { tr } \\
(\mathbf{m i n})\end{array}$ & $\mathbf{K I}_{\mathbf{E}}$ & $\mathbf{K I}_{\mathbf{R}}$ & \multicolumn{1}{|c|}{ Odor } \\
\hline A1 & Hexanal (44) & 9.31 & 1103.5 & 1108 & Fatty; fruity; green \\
\hline A2 & 1-Propanol 2-methyl (43) & 9.31 & 1103.0 & 1103 & Sweet; musty odor \\
\hline A3 & Pyridine (79) & 11.62 & 1205.5 & 1193 & Sour; fishy \\
\hline A4 & 1-Butanol 2-methyl (57) & 11.99 & 1221.5 & 1220 & Natural \\
\hline A5 & 1-Butanol 3-methyl (70) & 12.00 & 1222.0 & 1224 & $\begin{array}{l}\text { Fusel; alcohol; } \\
\text { sweet; fruity }\end{array}$ \\
\hline A6 & Limonene (68) & 12.00 & 1222.0 & 1221.5 & Herbaceous; minty \\
\hline A7 & 2-Hexenal (E) (41) & 12.50 & 1243.6 & 1238 & Green type flavor \\
\hline A8 & Cyclodecane methyl (55) & 12.82 & 1257.4 & 1260 & \\
\hline A9 & 2-Butanone 3-hydroxy (45) & 14.10 & 1313.1 & 1314 & Butter; creamy \\
\hline A10 & Tridecane (57) & 14.15 & 1315.3 & 1300 & Floral \\
\hline A11 & $\begin{array}{l}\text { 5-Hepten-2-one 6-methyl } \\
\text { (43) }\end{array}$ & 15.20 & 1361.8 & 1361 & $\begin{array}{l}\text { Oily; herbaceous; } \\
\text { green }\end{array}$ \\
\hline A12 & 1-Hexanol (56) & 15.37 & 1369.3 & 1362 & Herbal \\
\hline
\end{tabular}




\begin{tabular}{|c|c|c|c|c|c|}
\hline & & & & & Table $2 *$ (continue) \\
\hline Codes & Compounds & $\begin{array}{c}\operatorname{tr} \\
(\min )\end{array}$ & $\mathbf{K I}_{\mathbf{E}}$ & $\mathbf{K} \mathbf{I}_{\mathbf{R}}$ & Odor \\
\hline A13 & 3-Hexen-1-ol (Z) (41) & 16.14 & 1403.5 & 1406 & Green \\
\hline A14 & Nonanal (57) & 16.50 & 1420.1 & 1422 & $\begin{array}{l}\text { Apple; coconut; } \\
\text { grape; grapefruit; } \\
\text { lemon }\end{array}$ \\
\hline A15 & 2-Hexen-1-ol (E) (57) & 16.60 & 1424.8 & 1420 & $\begin{array}{l}\text { Apple; banana; } \\
\text { orange; green; } \\
\text { wine-like; }\end{array}$ \\
\hline A16 & Acetic acid (45) & 17.74 & 1477.4 & 1477 & $\begin{array}{l}\text { Pungent; sour; } \\
\text { vinegar-like odor }\end{array}$ \\
\hline A17 & Cycloisosativene (147) & 18.43 & 1509.8 & 1522 & \\
\hline A18 & n.i. (119) & 19.61 & 1566.9 & n.d. & \\
\hline A19 & Dimethyl sulfoxide (63) & 20.38 & 1604.3 & 1603 & $\begin{array}{l}\text { Butter; alliaceous } \\
\text { (onion; garlic) }\end{array}$ \\
\hline $\mathbf{A 2 0}$ & Alpha-Bergamotene (119) & 20.67 & 1619.1 & 1609 & Woody \\
\hline A21 & $\begin{array}{l}\text { Decanoic acid methyl ester } \\
(74)\end{array}$ & 20.74 & 1622.7 & 1624 & $\begin{array}{l}\text { Oily; fruity; wine- } \\
\text { like }\end{array}$ \\
\hline A22 & $\begin{array}{l}\text { Cycloheptanone 2-methylene } \\
(43)\end{array}$ & 20.98 & 1634.9 & n.d. & \\
\hline $\mathbf{A 2 3}$ & $\beta$-Farnesene (69) & 21.85 & 1679.2 & 1674 & $\begin{array}{l}\text { Apple; lavender; } \\
\text { lime; green; } \\
\text { woody; }\end{array}$ \\
\hline A24 & 8-Heptadecene (69) & 23.00 & 1739.6 & 1718 & \\
\hline A25 & Eremophilene (161) & 23.31 & 1756.2 & 1744 & \\
\hline A26 & $\alpha$-Farnesene (93) & 23.75 & 1779.7 & 1778 & Woody \\
\hline A27 & $\beta$-Sesquiphellandrene (69) & 24.30 & 1809.6 & 1782 & Herbal \\
\hline A28 & $\begin{array}{l}\text { Dodecanoic acid methyl ester } \\
(74)\end{array}$ & 24.73 & 1833.7 & 1834 & $\begin{array}{l}\text { Coconut; creamy; } \\
\text { soapy; waxy }\end{array}$ \\
\hline A29 & Hexanoic acid (60) & 25.50 & 1876.9 & 1874 & Cheese; fatty; sour \\
\hline A30 & Phenylethyl alcohol (91) & 26.85 & 1955.1 & 1946 & Honey; floral; rose \\
\hline A31 & $\begin{array}{l}\text { 2.6-Bis(1.1-dimethylethyl)-4- } \\
\text { (1-oxopropyl)phenol (233) }\end{array}$ & 26.90 & 1958.0 & n.d. & \\
\hline A32 & $\begin{array}{l}\text { 4.6-Heptadienoic acid. 3.3.6- } \\
\text { trimethyl methyl ester (109) }\end{array}$ & 27.12 & 1971.0 & n.d. & \\
\hline A33 & n.i. (159) & 27.29 & 1981.0 & n.d. & \\
\hline A34 & 3-Buten-2-ol. 2-methyl (71) & 27.49 & 1992.7 & n.d. & Herbal \\
\hline A35 & 1-Dodecanol (55) & 27.53 & 1995.1 & 1984 & $\begin{array}{l}\text { Coconut; honey; } \\
\text { soapy; waxy; } \\
\text { earthy; fatty }\end{array}$ \\
\hline A36 & Dimethylpalmitamine (58) & 28.03 & 2017.4 & n.d. & \\
\hline A37 & Methyl tetradecanoate (74) & 28.37 & 2031.7 & 2032 & Honey; fatty \\
\hline
\end{tabular}




\begin{tabular}{|c|c|c|c|c|c|}
\hline & & & & & Table $2 *$ (continue) \\
\hline Codes & Compounds & $\begin{array}{c}\operatorname{tr} \\
(\min )\end{array}$ & $\mathbf{K} \mathbf{I}_{\mathbf{E}}$ & $\mathbf{K} \mathbf{I}_{\mathbf{R}}$ & Odor \\
\hline A38 & Phenol (94) & 28.45 & 2035.0 & 2035 & Sweet; tarry odor \\
\hline A39 & Nerolidol 2 (69) & 28.86 & 2052.2 & 2052 & $\begin{array}{l}\text { Apple; green; } \\
\text { woody; citrus; rose }\end{array}$ \\
\hline A40 & $\begin{array}{l}2(3 \mathrm{H}) \text {-Furanone dihydro-5- } \\
\text { propyl }(\gamma \text {-lactone }(85)\end{array}$ & 29.10 & 2062.3 & 2068 & $\begin{array}{l}\text { Waxy; creamy; } \\
\text { coconut character; }\end{array}$ \\
\hline A41 & $\begin{array}{l}\text { Benzoic acid 2-methoxy. } \\
\text { methyl ester (135) }\end{array}$ & 29.69 & 2087.0 & 2088 & \\
\hline A42 & $\begin{array}{l}\text { 2H-Pyran-2-one. 6- } \\
\text { hexyltetrahydro (99) }\end{array}$ & 32.70 & 2213.2 & 2215 & Fruity; sweet \\
\hline A43 & $\begin{array}{l}\text { Hexadecanoic acid methyl } \\
\text { ester (74) }\end{array}$ & 32.75 & 2215.3 & 2214 & Waxy \\
\hline A44 & n.i. (71) & 33.80 & 2259.3 & n.d. & \\
\hline A45 & $\begin{array}{l}\text { 9-Octadecenoic acid methyl } \\
\text { ester (oleic acid) (55) }\end{array}$ & 37.51 & 2414.8 & 2424 & Fatty \\
\hline A46 & p-Isopropenylphenol (134) & 37.76 & 2425.2 & n.d. & \\
\hline
\end{tabular}

* KI $\mathrm{E}_{\mathrm{E}}$ experiment value of Kovats index (KI);

$* \mathrm{KI}$. reference value of $\mathrm{KI}$.

*The odor descriptors were obtained from SAFC "Flavors and Fragrances. European Ed. Catalogue 2009". 
Table 3*

Normalized peak area of volatile compounds isolated from Edremit (Ayvalık) oils in two different packaging at initial and after 7, 14 and 21 days of ripening

\begin{tabular}{|c|c|c|c|c|c|c|c|c|}
\hline \multirow{3}{*}{$\frac{\mathscr{e}}{0}$} & \multirow{3}{*}{$\begin{array}{l}\text { Compounds } \\
\qquad(\mathbf{m} / \mathbf{z})\end{array}$} & \multicolumn{7}{|c|}{ Normalised peak area $($ mean \pm SD. $n=2)$} \\
\hline & & \multirow[b]{2}{*}{$\mathbf{E}_{\mathbf{0}}$} & \multicolumn{3}{|c|}{$\mathbf{E}_{\mathbf{C}}$} & \multicolumn{3}{|c|}{$\mathbf{E}_{\mathbf{K}}$} \\
\hline & & & $\mathbf{E}_{7 \mathrm{C}}$ & $\mathbf{E}_{14 \mathrm{C}}$ & $\mathbf{E}_{21 \mathrm{C}}$ & $\mathbf{E}_{7 \mathbf{K}}$ & $\mathbf{E}_{14 \mathrm{~K}}$ & $\mathbf{E}_{21 \mathrm{~K}}$ \\
\hline A1 & Hexanal (44) & $(251 \pm 11)^{\mathrm{a}}$ & $\begin{array}{c}(29.74 \pm \\
0.14)^{\mathrm{b}}\end{array}$ & n.d. ${ }^{\mathrm{c}}$ & n.d. ${ }^{c}$ & $(9 \pm 2)^{\mathrm{c}}$ & n.d. ${ }^{\mathrm{c}}$ & n.d. ${ }^{c}$ \\
\hline A2 & $\begin{array}{l}\text { 1-Propanol 2-methyl } \\
\text { (43) }\end{array}$ & n.d. ${ }^{b}$ & n.d. ${ }^{b}$ & $(9 \pm 2)^{\mathrm{a}}$ & $\begin{array}{c}(10.6 \pm \\
0.3)^{\mathrm{a}} \\
\end{array}$ & n.d. ${ }^{b}$ & n.d. ${ }^{b}$ & n.d. ${ }^{b}$ \\
\hline A3 & Pyridine (79) & n.d. ${ }^{b}$ & $\begin{array}{c}(32.7 \pm \\
0.4)^{\mathrm{a}}\end{array}$ & n.d. ${ }^{b}$ & n.d. ${ }^{b}$ & n.d. ${ }^{b}$ & n.d. ${ }^{b}$ & n.d. ${ }^{b}$ \\
\hline A4 & $\begin{array}{l}\text { 1-Butanol 2-methyl } \\
\text { (57) }\end{array}$ & n.d. ${ }^{\mathrm{c}}$ & $\begin{array}{l}(1.7 \pm \\
0.2)^{\mathrm{bc}} \\
\end{array}$ & $\begin{array}{l}(4.0 \pm \\
1.0)^{\mathrm{a}} \\
\end{array}$ & $\begin{array}{c}(5.0 \pm \\
0.7)^{\mathrm{a}} \\
\end{array}$ & $\begin{array}{l}(1.32 \pm \\
0.10)^{\mathrm{bc}}\end{array}$ & $\begin{array}{l}(1.43 \pm \\
0.04)^{\mathrm{bc}}\end{array}$ & $\begin{array}{l}(1.9 \pm \\
0.2)^{\mathrm{b}} \\
\end{array}$ \\
\hline A5 & $\begin{array}{l}\text { 1-Butanol 3-methyl } \\
\text { (70) }\end{array}$ & n.d. ${ }^{\mathrm{e}}$ & $\begin{array}{l}(1.8 \pm \\
0.3)^{\mathrm{cd}}\end{array}$ & $\begin{array}{l}(4.0 \pm \\
0.2)^{\mathrm{b}}\end{array}$ & $\begin{array}{c}5.59 \\
\pm 0.04)^{\mathrm{a}} \\
\end{array}$ & $\begin{array}{c}(1.24 \\
\pm 0.08)^{\mathrm{d}} \\
\end{array}$ & $\begin{array}{c}(1.73 \\
\pm 0.11)^{\mathrm{cd}}\end{array}$ & $\begin{array}{c}(2.06 \\
\pm 0.09)^{\mathrm{c}} \\
\end{array}$ \\
\hline A6 & Limonene (68) & $\begin{array}{l}(2.70 \pm \\
0.06)^{\mathrm{bc}}\end{array}$ & $\begin{array}{c}(40.2 \pm \\
0.4)^{\mathrm{a}} \\
\end{array}$ & $\begin{array}{l}(1.95 \pm \\
0.12)^{\mathrm{cd}}\end{array}$ & $\begin{array}{l}(0.80 \pm \\
0.07)^{\mathrm{e}} \\
\end{array}$ & $\begin{array}{l}(2.8 \pm \\
0.2)^{\mathrm{b}}\end{array}$ & n.d. ${ }^{\mathrm{f}}$ & $\begin{array}{l}(1.9 \pm \\
0.2)^{\mathrm{d}} \\
\end{array}$ \\
\hline A7 & 2-Hexenal (E) (41) & $\begin{array}{c}(14.0 \pm \\
1.3)^{\mathrm{a}}\end{array}$ & $\begin{array}{l}(0.43 \pm \\
0.09)^{b}\end{array}$ & $\begin{array}{l}(0.82 \pm \\
0.03)^{\mathrm{b}}\end{array}$ & n.d. ${ }^{b}$ & n.d. ${ }^{b}$ & n.d. ${ }^{b}$ & n.d. ${ }^{b}$ \\
\hline A8 & $\begin{array}{l}\text { Cyclodecane methyl } \\
(55)\end{array}$ & $\begin{array}{c}(12.25+ \\
0.12)^{\mathrm{d}} \\
\end{array}$ & $\begin{array}{c}(17.66 \pm \\
0.07)^{\mathrm{c}} \\
\end{array}$ & $\begin{array}{c}(22.8 \pm \\
0.6)^{\mathrm{a}} \\
\end{array}$ & $\begin{array}{l}21.6 \pm \\
1.3)^{\mathrm{ab}} \\
\end{array}$ & $\begin{array}{l}(19.3 \pm \\
0.14)^{\mathrm{bc}}\end{array}$ & $\begin{array}{l}(9.6 \pm \\
0.5)^{\mathrm{e}} \\
\end{array}$ & $\begin{array}{c}(17.4 \pm \\
0.8)^{\mathrm{d}} \\
\end{array}$ \\
\hline A9 & $\begin{array}{l}\text { 2-Butanone 3- } \\
\text { hydroxy (45) }\end{array}$ & n.d. ${ }^{b}$ & n.d. ${ }^{b}$ & \begin{tabular}{|l}
$(5.22 \pm$ \\
$0.03)^{\mathrm{a}}$ \\
\end{tabular} & \begin{tabular}{|l}
$(5.85 \pm$ \\
$0.13)^{\mathrm{a}}$ \\
\end{tabular} & n.d. ${ }^{b}$ & $\begin{array}{l}(1.7 \pm \\
0.5)^{\mathrm{b}} \\
\end{array}$ & $\begin{array}{l}(1.8 \pm \\
1.1)^{\mathrm{b}} \\
\end{array}$ \\
\hline A10 & Tridecane (57) & $\begin{array}{l}(2.20 \pm \\
0.04)^{\mathrm{a}} \\
\end{array}$ & $\begin{array}{l}(2.0 \pm \\
0.2)^{\mathrm{a}} \\
\end{array}$ & $\begin{array}{l}(1.9 \pm \\
0.4)^{\mathrm{a}} \\
\end{array}$ & $\begin{array}{l}(2.9 \pm \\
0.6)^{\mathrm{a}} \\
\end{array}$ & $\begin{array}{l}(2.51 \pm \\
0.11)^{\mathrm{a}} \\
\end{array}$ & $\begin{array}{l}(1.9 \pm \\
1.0)^{\mathrm{a}} \\
\end{array}$ & $\begin{array}{l}(1.7 \pm \\
0.5)^{\mathrm{a}} \\
\end{array}$ \\
\hline A11 & $\begin{array}{l}\text { 5-Hepten-2-one. 6- } \\
\text { methyl (43) }\end{array}$ & n.d. ${ }^{b}$ & $\begin{array}{l}(2.0 \pm \\
0.3)^{\mathrm{ab}}\end{array}$ & $\begin{array}{l}(1.2 \pm \\
0.3)^{\mathrm{ab}} \\
\end{array}$ & $\begin{array}{l}(2.6 \pm \\
0.4)^{\mathrm{a}} \\
\end{array}$ & $\begin{array}{l}(0.42 \pm \\
0.11)^{\mathrm{b}} \\
\end{array}$ & $\begin{array}{c}(0.48 \\
\pm 0.02)^{\mathrm{b}} \\
\end{array}$ & $\begin{array}{l}(1.19 \pm \\
0.02)^{\mathrm{ab}} \\
\end{array}$ \\
\hline A12 & 1-Hexanol (56) & $(33 \pm 2)^{f}$ & $(236 \pm 9)^{d}$ & $\begin{array}{c}(503 \pm \\
11)^{\mathrm{a}} \\
\end{array}$ & $\begin{array}{c}(268 \pm \\
3)^{\mathrm{c}} \\
\end{array}$ & $\begin{array}{c}(356 \pm \\
4)^{b}\end{array}$ & $(119 \pm 13)^{\mathrm{e}}$ & $\begin{array}{c}(144.3 \pm \\
0.2)^{\mathrm{e}}\end{array}$ \\
\hline A13 & $\begin{array}{l}\text { 3-Hexen-1-ol (Z) } \\
(41)\end{array}$ & $\begin{array}{c}(13.87 \pm \\
0.11)^{\mathrm{e}} \\
\end{array}$ & $(52 \pm 2)^{b}$ & $\begin{array}{c}60.5 \pm \\
1.4)^{\mathrm{a}} \\
\end{array}$ & $\begin{array}{l}(51.9 \pm \\
0.8)^{\mathrm{b}} \\
\end{array}$ & $\begin{array}{l}(49.4 \pm \\
0.9)^{\mathrm{bc}}\end{array}$ & $(30 \pm 3)^{\mathrm{d}}$ & $\begin{array}{c}(44.1 \pm \\
0.4)^{\mathrm{c}} \\
\end{array}$ \\
\hline A14 & Nonanal (57) & $\begin{array}{l}(8.7 \pm \\
1.5)^{\mathrm{b}} \\
\end{array}$ & $\begin{array}{l}(3.0 \pm \\
0.4)^{\mathrm{bc}}\end{array}$ & $\begin{array}{c}(54.9 \pm \\
1.4)^{\mathrm{a}}\end{array}$ & $\begin{array}{l}(3.9 \pm \\
0.4)^{\mathrm{bc}}\end{array}$ & $\begin{array}{l}(4.32 \pm \\
2.03)^{\mathrm{bc}}\end{array}$ & $\begin{array}{l}(1.6 \pm \\
0.8)^{\mathrm{c}}\end{array}$ & n.d. ${ }^{c}$ \\
\hline A15 & $\begin{array}{l}\text { 2-Hexen-1-ol (E) } \\
(57)\end{array}$ & n.d. ${ }^{d}$ & $\begin{array}{c}(19.3 \pm \\
0.5)^{\mathrm{c}} \\
\end{array}$ & $\begin{array}{c}(53.6 \pm \\
0.5)^{\mathrm{a}} \\
\end{array}$ & n.d. ${ }^{\mathrm{d}}$ & $(35 \pm 3)^{b}$ & n.d. ${ }^{\mathrm{d}}$ & $\begin{array}{c}(18.2 \pm \\
0.7)^{\mathrm{c}} \\
\end{array}$ \\
\hline A16 & Acetic acid (45) & $\begin{array}{c}(14.1 \pm \\
0.2)^{\mathrm{a}} \\
\end{array}$ & $\begin{array}{c}(10.5 \pm \\
1.1)^{\mathrm{a}} \\
\end{array}$ & $(11 \pm 2)^{\mathrm{a}}$ & $(12 \pm 2)^{a}$ & $(8 \pm 2)^{\mathrm{a}}$ & $(7 \pm 6)^{\mathrm{a}}$ & $\begin{array}{c}(7.5 \pm \\
0.7)^{\mathrm{a}} \\
\end{array}$ \\
\hline A17 & $\begin{array}{l}\text { Cycloisosativene } \\
\text { (147) }\end{array}$ & n.d. ${ }^{a}$ & n.d. ${ }^{a}$ & n.d. ${ }^{a}$ & n.d. ${ }^{a}$ & n.d. ${ }^{a}$ & n.d. ${ }^{a}$ & n.d. ${ }^{\text {a }}$ \\
\hline A18 & n.i. (119) & $\begin{array}{l}(3.0 \pm \\
0.3)^{\mathrm{a}} \\
\end{array}$ & $\begin{array}{l}(2.0 \pm \\
0.7)^{\mathrm{a}} \\
\end{array}$ & \begin{tabular}{|c|}
$(4.41 \pm$ \\
$0.12)^{\mathrm{a}}$ \\
\end{tabular} & $\begin{array}{r}(4.5 \pm \\
0.2)^{\mathrm{a}} \\
\end{array}$ & $\begin{array}{l}(6.2 \pm \\
1.5)^{\mathrm{a}} \\
\end{array}$ & $\begin{array}{c}(4.2 \pm \\
0.2)^{\mathrm{a}} \\
\end{array}$ & $\begin{array}{l}(5.8 \pm \\
0.2)^{\mathrm{a}} \\
\end{array}$ \\
\hline A19 & $\begin{array}{l}\text { Dimethyl sulfoxide } \\
\text { (63) }\end{array}$ & $\begin{array}{c}(3.0 \pm \\
0.2)^{\mathrm{a}} \\
\end{array}$ & $\begin{array}{l}(0.8 \pm \\
0.3)^{\mathrm{b}} \\
\end{array}$ & \begin{tabular}{|l}
$(1.18 \pm$ \\
$0.01)^{\mathrm{b}}$ \\
\end{tabular} & $\begin{array}{c}(3.7 \pm \\
0.7)^{\mathrm{a}} \\
\end{array}$ & $\begin{array}{l}(0.5 \pm \\
0.2)^{b} \\
\end{array}$ & $\begin{array}{l}(0.75 \pm \\
0.14)^{\mathrm{b}} \\
\end{array}$ & $\begin{array}{l}(0.74 \pm \\
0.04)^{b} \\
\end{array}$ \\
\hline A20 & $\alpha$-Bergamotene (119) & $\begin{array}{l}(1.99 \pm \\
0.01)^{\mathrm{b}}\end{array}$ & $\begin{array}{l}(2.3 \pm \\
0.3)^{\mathrm{b}}\end{array}$ & $\begin{array}{l}(2.32 \pm \\
0.01)^{b} \\
\end{array}$ & $\begin{array}{l}(2.25 \pm \\
0.12)^{\mathrm{b}} \\
\end{array}$ & $\begin{array}{l}(4.2 \pm \\
0.9)^{\mathrm{a}} \\
\end{array}$ & $\begin{array}{c}(1.63 \\
\pm 0.05)^{\mathrm{b}}\end{array}$ & $\begin{array}{l}(3.0 \pm \\
0.2)^{\mathrm{ab}} \\
\end{array}$ \\
\hline A21 & $\begin{array}{l}\text { Decanoic acid methyl } \\
\text { ester (74) }\end{array}$ & n.d. ${ }^{b}$ & n.d. ${ }^{b}$ & n.d. ${ }^{b}$ & $\begin{array}{c}(31.5 \pm \\
0.9)^{\mathrm{a}}\end{array}$ & n.d. ${ }^{b}$ & n.d. ${ }^{\text {b }}$ & n.d. ${ }^{b}$ \\
\hline A22 & $\begin{array}{l}\text { Cycloheptanone 2- } \\
\text { methylene (43) }\end{array}$ & n.d. ${ }^{\mathrm{c}}$ & n.d. ${ }^{c}$ & $\begin{array}{l}(3.1 \pm \\
0.4)^{b}\end{array}$ & $\begin{array}{l}(5.1 \pm \\
0.2)^{\mathrm{a}} \\
\end{array}$ & n.d. ${ }^{\mathrm{c}}$ & n.d. ${ }^{c}$ & n.d. ${ }^{c}$ \\
\hline
\end{tabular}




\begin{tabular}{|c|c|c|c|c|c|c|c|c|}
\hline & & & & & & & Table3* & (continue) \\
\hline$\infty$ & & & Norma & alised pe: & ak area & (mean= & \pm SD. $n=2)$ & \\
\hline$\frac{0}{0}$ & Compounds (m/z) & & & $\mathbf{E}_{\mathbf{C}}$ & & & $\mathbf{E}_{\mathbf{K}}$ & \\
\hline ن & & $\mathbf{E}_{0}$ & $\mathbf{E}_{7 \mathrm{C}}$ & $\mathbf{E}_{14 \mathrm{C}}$ & $\mathbf{E}_{21 C}$ & $\mathbf{E}_{7 \mathrm{~K}}$ & $\mathbf{E}_{14 K}$ & $\mathbf{E}_{21 K}$ \\
\hline A23 & (E)- $\beta$-Fanesene (69) & $\begin{array}{l}(9.1 \pm \\
0.3)^{\mathrm{b}}\end{array}$ & $\begin{array}{c}(10.2 \pm \\
0.5)^{\mathrm{b}}\end{array}$ & $\begin{array}{l}(11.2 \pm \\
0.3)^{\mathrm{b}}\end{array}$ & $\begin{array}{l}(12.1 \pm \\
1.0)^{\mathrm{ab}}\end{array}$ & $(19 \pm 5)^{\mathrm{a}}$ & $\begin{array}{l}(7.4 \pm \\
0.2)^{\mathrm{b}}\end{array}$ & $\begin{array}{l}(14.0 \pm \\
0.4)^{\mathrm{ab}}\end{array}$ \\
\hline A24 & 8-Heptadecene (69) & n.d. ${ }^{\mathrm{a}}$ & n.d. ${ }^{\mathrm{a}}$ & n.d. ${ }^{\mathrm{a}}$ & n.d. $^{\mathrm{a}}$ & n.d. $^{a}$ & n.d. ${ }^{\mathrm{a}}$ & n.d. ${ }^{\mathrm{a}}$ \\
\hline A25 & Eremophilene (161) & $\begin{array}{l}(0.79 \pm \\
0.03)^{\mathrm{d}}\end{array}$ & $\begin{array}{l}(2.62 \pm \\
0.03)^{\mathrm{bc}}\end{array}$ & $\begin{array}{l}(5.3 \pm \\
0.4)^{\mathrm{a}}\end{array}$ & $\begin{array}{l}(5.0 \pm \\
0.2)^{\mathrm{a}}\end{array}$ & $\begin{array}{l}(3.7 \pm \\
1.0)^{\mathrm{abc}}\end{array}$ & $\begin{array}{l}(2.02 \pm \\
0.07)^{\mathrm{cd}}\end{array}$ & $\begin{array}{l}(3.8 \pm \\
0.2)^{\mathrm{ab}}\end{array}$ \\
\hline A26 & $\alpha$-Farnesene (93) & $\begin{array}{c}(150 \pm \\
20)^{\mathrm{a}}\end{array}$ & $\begin{array}{l}(88 \pm \\
2)^{\text {bcd }}\end{array}$ & $\begin{array}{c}(136 \pm \\
8)^{\mathrm{ab}}\end{array}$ & $\begin{array}{c}(120 \pm \\
4)^{\mathrm{abc}}\end{array}$ & $\begin{array}{l}(110 \pm \\
40)^{\mathrm{abc}}\end{array}$ & $\begin{array}{c}(41 \pm \\
2)^{\mathrm{d}}\end{array}$ & $\begin{array}{c}(62.8 \pm \\
0.2)^{\mathrm{cd}}\end{array}$ \\
\hline A27 & $\begin{array}{c}\beta \text {-Sesquiphellandrene } \\
\text { (69) }\end{array}$ & $\begin{array}{l}(8.7 \pm \\
1.0)^{\mathrm{ab}}\end{array}$ & $\begin{array}{l}(8.3 \pm \\
0.6)^{\mathrm{ab}}\end{array}$ & $\begin{array}{l}(7.3 \pm \\
0.6)^{\mathrm{ab}}\end{array}$ & $\begin{array}{l}(8.20 \pm \\
0.14)^{\mathrm{ab}}\end{array}$ & $\begin{array}{c}(14 \pm \\
5)^{\mathrm{a}}\end{array}$ & $\begin{array}{l}(4.5 \pm \\
0.2)^{b}\end{array}$ & $\begin{array}{l}(8.3 \pm \\
0.4)^{\mathrm{ab}}\end{array}$ \\
\hline A28 & $\begin{array}{l}\text { Dodecanoic acid } \\
\text { methyl ester (74) }\end{array}$ & $\begin{array}{c}\text { n.d. } \\
\text { b }\end{array}$ & $\underset{\substack{\text { n.d. } \\
\mathrm{b}}}{\mathrm{n}}$ & $\underset{\substack{\text { n.d. } \\
\mathrm{b}}}{\mathrm{n}}$ & $\begin{array}{c}(27.0 \pm \\
0.3)^{\mathrm{a}}\end{array}$ & $\begin{array}{c}\text { n.d. } \\
\mathrm{b}\end{array}$ & $\begin{array}{l}(0.8 \pm \\
0.3)^{\mathrm{b}}\end{array}$ & $\begin{array}{c}(0.43 \\
\pm 0.2)^{0 \mathrm{~b}}\end{array}$ \\
\hline A29 & Hexanoic acid (60) & $\begin{array}{c}(17 \pm \\
6)^{\mathrm{a}}\end{array}$ & $\begin{array}{l}(10 \pm \\
2)^{\mathrm{ab}}\end{array}$ & $\begin{array}{c}(16.9 \pm \\
1.3)^{\mathrm{a}}\end{array}$ & $\begin{array}{c}(20.9 \pm \\
0.2)^{\mathrm{a}}\end{array}$ & $\begin{array}{l}(4 \pm \\
2)^{b}\end{array}$ & $\begin{array}{l}(1.7 \pm \\
1.0)^{\mathrm{a}}\end{array}$ & $\begin{array}{l}(3 \pm \\
2)^{\mathrm{b}}\end{array}$ \\
\hline A30 & $\begin{array}{l}\text { Phenylethyl alcohol } \\
\text { (91) }\end{array}$ & $(5 \pm 3)^{\mathrm{c}}$ & $(12 \pm 5)^{\mathrm{abc}}$ & $(18 \pm 5)^{\mathrm{ab}}$ & $\begin{array}{c}(19.6 \pm \\
0.7)^{\mathrm{a}} \\
\end{array}$ & $\begin{array}{l}(9.4 \pm \\
0.3)^{\mathrm{abc}} \\
\end{array}$ & $\begin{array}{l}(4.9 \pm \\
0.8)^{\mathrm{c}} \\
\end{array}$ & $\begin{array}{l}(7.1 \pm \\
0.4)^{\mathrm{bc}}\end{array}$ \\
\hline A31 & $\begin{array}{c}2.6-\operatorname{Bis}(1.1- \\
\text { dimethylethyl)-4-(1- } \\
\text { oxopropyl) phenol } \\
(233) \\
\end{array}$ & $(15 \pm 7)^{\mathrm{a}}$ & $\begin{array}{l}(9.32 \pm \\
0.08)^{\mathrm{ab}}\end{array}$ & $\begin{array}{c}(10.4 \pm \\
0.5)^{\mathrm{ab}}\end{array}$ & $\begin{array}{l}(10.7 \pm \\
0.8)^{\mathrm{ab}}\end{array}$ & $\begin{array}{l}(9.74 \pm \\
0.05)^{\mathrm{ab}}\end{array}$ & $\begin{array}{l}(0.9 \pm \\
0.2)^{b}\end{array}$ & $\begin{array}{l}(10.1 \pm \\
0.5)^{\mathrm{ab}}\end{array}$ \\
\hline A32 & $\begin{array}{c}\text { 4.6-Heptadienoic acid } \\
\text { 3.3.6-trimethyl. methyl } \\
\text { ester (109) }\end{array}$ & n.d. ${ }^{\mathrm{a}}$ & $\begin{array}{l}(2.1 \pm \\
1.1)^{\mathrm{a}}\end{array}$ & $\begin{array}{l}(20 \pm \\
15)^{\mathrm{a}}\end{array}$ & $\begin{array}{r}(1.4 \pm \\
0.6)^{\mathrm{a}}\end{array}$ & $(7 \pm 5)^{\mathrm{a}}$ & n.d. ${ }^{\mathrm{a}}$ & $(1.6 \pm 0.9)^{\mathrm{a}}$ \\
\hline $\mathbf{A 3 3}$ & n.i. (159) & n.d. ${ }^{\mathrm{c}}$ & $\begin{array}{l}(0.96 \pm \\
0.05)^{\mathrm{bc}}\end{array}$ & $\begin{array}{l}(1.2 \pm \\
0.4)^{\mathrm{ab}}\end{array}$ & $\begin{array}{l}(1.65 \pm \\
0.14)^{\mathrm{a}}\end{array}$ & n.d. ${ }^{\mathrm{c}}$ & n.d. ${ }^{\mathrm{c}}$ & n.d. ${ }^{\mathrm{c}}$ \\
\hline A34 & $\begin{array}{c}\text { 3-Buten-2-ol 2-methyl } \\
\text { (71) }\end{array}$ & n.d. ${ }^{b}$ & $(6 \pm 5)^{\mathrm{ab}}$ & $\begin{array}{l}(11 \pm \\
7)^{\mathrm{ab}}\end{array}$ & $\begin{array}{l}(3.1 \pm \\
0.5)^{\mathrm{ab}} \\
\end{array}$ & \begin{tabular}{|l}
$(13.1 \pm$ \\
$0.9)^{\mathrm{a}}$ \\
\end{tabular} & $\begin{array}{c}(1.43 \\
0.07)^{\mathrm{ab}}\end{array}$ & $\begin{array}{l}(2.8 \pm \\
0.3)^{\mathrm{ab}}\end{array}$ \\
\hline A35 & 1-Dodecanol (55) & $(8 \pm 5)^{\mathrm{a}}$ & n.d. ${ }^{\mathrm{a}}$ & $\begin{array}{l}(1.5 \pm \\
0.3)^{\mathrm{a}}\end{array}$ & n.d. ${ }^{a}$ & $\begin{array}{c}(10 \pm \\
4)^{\mathrm{a}} \\
\end{array}$ & n.d. ${ }^{a}$ & $\begin{array}{c}(0.8 \pm \\
0.5)^{\mathrm{a}} \\
\end{array}$ \\
\hline A36 & \begin{tabular}{|c|} 
Dimethylpalmitamine \\
$(58)$
\end{tabular} & $(80 \pm 70)^{\mathrm{a}}$ & n.d. ${ }^{\mathrm{a}}$ & n.d. ${ }^{a}$ & n.d. ${ }^{\mathrm{a}}$ & $(5 \pm 4)^{\mathrm{a}}$ & n.d. ${ }^{\mathrm{a}}$ & n.d. ${ }^{\mathrm{a}}$ \\
\hline A37 & $\begin{array}{c}\text { Methyl tetradecanoate } \\
\text { (74) }\end{array}$ & n.d. ${ }^{b}$ & n.d. ${ }^{b}$ & n.d. ${ }^{b}$ & $(27 \pm 2)^{\mathrm{a}}$ & n.d. ${ }^{b}$ & n.d. ${ }^{b}$ & n.d. ${ }^{b}$ \\
\hline A38 & Phenol (94) & $\begin{array}{l}(2.8 \pm \\
0.9)^{\mathrm{a}}\end{array}$ & $\begin{array}{l}(2.3 \pm \\
0.2)^{\mathrm{a}}\end{array}$ & $\begin{array}{l}(2.66 \pm \\
0.10)^{\mathrm{a}}\end{array}$ & $\begin{array}{l}(3.44 \pm \\
0.09)^{\mathrm{a}}\end{array}$ & $\begin{array}{l}(2.4 \pm \\
0.2)^{\mathrm{a}}\end{array}$ & $(3.5 \pm 0.4)^{\mathrm{a}}$ & $\begin{array}{l}(2.21 \pm \\
0.02)^{\mathrm{a}}\end{array}$ \\
\hline A39 & Nerolidol 2 (69) & n.d. ${ }^{a}$ & n.d. ${ }^{a}$ & n.d. ${ }^{\mathrm{a}}$ & n.d. $^{\mathrm{a}}$ & n.d. $^{\mathrm{a}}$ & n.d. ${ }^{\mathrm{a}}$ & n.d. ${ }^{\mathrm{a}}$ \\
\hline A40 & \begin{tabular}{|c|} 
2(3H)-Furanone. \\
dihydro-5-propyl (85)
\end{tabular} & $\begin{array}{l}(3.1 \pm \\
0.8)^{\mathrm{b}}\end{array}$ & $\begin{array}{l}(4.0 \pm \\
1.2)^{\mathrm{b}}\end{array}$ & $\begin{array}{l}(7.0 \pm \\
0.5)^{\mathrm{a}}\end{array}$ & \begin{tabular}{|l|}
$(8.65 \pm$ \\
$0.05)^{\mathrm{a}}$ \\
\end{tabular} & $\begin{array}{l}(4.14 \pm \\
0.13)^{\mathrm{b}}\end{array}$ & $(2.8 \pm 0.3)^{\mathrm{b}}$ & $(3.2 \pm 0.3)^{\mathrm{b}}$ \\
\hline A41 & $\begin{array}{c}\text { Benzoic acid. 2- } \\
\text { methoxy. methyl ester } \\
\text { (135) }\end{array}$ & $(5 \pm 2)^{\mathrm{a}}$ & $\begin{array}{l}(1.9 \pm \\
0.6)^{\mathrm{ab}}\end{array}$ & $\begin{array}{l}(2.10 \pm \\
0.09)^{\mathrm{ab}}\end{array}$ & $\begin{array}{l}(1.3 \pm \\
0.4)^{\mathrm{ab}}\end{array}$ & $\begin{array}{l}(2.5 \pm \\
0.3)^{\mathrm{ab}}\end{array}$ & n.d. ${ }^{b}$ & $\begin{array}{l}(0.96 \pm \\
0.05)^{\mathrm{ab}}\end{array}$ \\
\hline
\end{tabular}




\begin{tabular}{|c|c|c|c|c|c|c|c|c|}
\hline \multicolumn{9}{|c|}{ Table $3 *$ (continue) } \\
\hline \multirow{3}{*}{$\frac{8}{\tilde{g}}$} & \multirow{3}{*}{ Compounds $(\mathbf{m} / \mathbf{z})$} & \multicolumn{7}{|c|}{ Normalised peak area $($ mean \pm SD. $n=2)$} \\
\hline & & & \multicolumn{3}{|c|}{$\mathbf{E}_{\mathbf{C}}$} & \multicolumn{3}{|c|}{$\mathbf{E}_{\mathbf{K}}$} \\
\hline & & $\mathbf{E}_{0}$ & E7C $_{7}$ & $\mathbf{E}_{14 \mathrm{C}}$ & $\mathbf{E}_{21 C}$ & $\mathbf{E}_{7 \mathbf{K}}$ & $\mathbf{E}_{\mathbf{1 4 K}}$ & $\mathbf{E}_{21 K}$ \\
\hline A42 & $\begin{array}{l}\text { 2H-Pyran-2-one. 6- } \\
\text { hexyltetrahydro (99) }\end{array}$ & $(4 \pm 2)^{\mathrm{a}}$ & $(5 \pm 3)^{\mathrm{a}}$ & $(3 \pm 2)^{\mathrm{a}}$ & $\begin{array}{l}(2.0 \pm \\
0.2)^{\mathrm{a}}\end{array}$ & $\begin{array}{l}(2.0 \pm \\
0.7)^{\mathrm{a}}\end{array}$ & n.d. ${ }^{a}$ & n.d. ${ }^{a}$ \\
\hline A43 & $\begin{array}{c}\text { Hexadecanoic acid methyl } \\
\text { ester (74) }\end{array}$ & $(9 \pm 4)^{b}$ & $(5 \pm 4)^{b}$ & $(12 \pm 2)^{b}$ & $(33 \pm 5)^{\mathrm{a}}$ & $(5 \pm 4)^{\mathrm{b}}$ & $\begin{array}{l}(5.0 \pm \\
0.3)^{b}\end{array}$ & $(6 \pm 2)^{b}$ \\
\hline A44 & n.i. (71) & $(1.3 \pm 0.4)^{\mathrm{a}}$ & $\begin{array}{c}(12 \pm \\
10)^{\mathrm{a}}\end{array}$ & $\begin{array}{l}(30 \pm \\
20)^{\mathrm{a}}\end{array}$ & $\begin{array}{c}(14.9 \\
\pm 1.3)^{\mathrm{a}}\end{array}$ & $(5 \pm 3)^{\mathrm{a}}$ & n.d. ${ }^{a}$ & $(2.2 \pm 0.2)^{\mathrm{a}}$ \\
\hline A45 & $\begin{array}{l}\text { 9-Octadecenoic acid methyl } \\
\text { ester (55) }\end{array}$ & $\begin{array}{l}(5.77 \pm \\
0.08)^{\mathrm{b}}\end{array}$ & $(4 \pm 3)^{b}$ & $\begin{array}{c}(18.0 \pm \\
0.3)^{\mathrm{a}}\end{array}$ & $\begin{array}{c}(15 \pm \\
5)^{\mathrm{ab}}\end{array}$ & $(6 \pm 7)^{b}$ & $\begin{array}{l}(4.5 \pm \\
0.7)^{b}\end{array}$ & $(8 \pm 2)^{b}$ \\
\hline A46 & p-Isopropenylphenol (134) & $\begin{array}{l}(1.7 \pm \\
0.4)^{\mathrm{b}}\end{array}$ & $\begin{array}{l}(1.6 \pm \\
0.8)^{\mathrm{b}}\end{array}$ & $\begin{array}{l}(2.2 \pm \\
0.8)^{\mathrm{b}}\end{array}$ & $\begin{array}{l}(1.8 \pm \\
0.3)^{b}\end{array}$ & $\begin{array}{l}(2.4 \pm \\
0.3)^{\mathrm{b}}\end{array}$ & $\begin{array}{c}(11 \pm \\
3)^{\mathrm{a}}\end{array}$ & $\begin{array}{l}(2.40 \pm \\
0.09)^{\mathrm{b}}\end{array}$ \\
\hline
\end{tabular}

${ }^{*}$ Peak areas are normalised respect to internal standard ${ }^{1}$ n.d. not detected.

${ }^{\text {a-f }}$ Values in the same row with different superscript letters differ significantly $(p<0.05)$.

Data showed mean of two independent tests (mean \pm sd). 
Table 4*

Volatile compounds $\left(\mathrm{AU} \times 10^{4}\right)$ isolated from Uslu oils in two different packaging at initial and after 7, 14 and 21 days of stages of ripening

\begin{tabular}{|c|c|c|c|c|c|c|c|c|}
\hline \multirow{3}{*}{ ن் } & \multirow{3}{*}{$\begin{array}{c}\text { Compounds } \\
(\mathbf{m} / \mathbf{z})\end{array}$} & \multicolumn{7}{|c|}{ Normalised peak area (mean \pm SD. $n=2)$} \\
\hline & & & \multicolumn{3}{|c|}{$\mathbf{U}_{\mathbf{C}}$} & \multicolumn{3}{|c|}{$\mathbf{U}_{\mathbf{K}}$} \\
\hline & & $\mathbf{U}_{0}$ & $\mathbf{U}_{7 \mathrm{C}}$ & $\mathbf{U}_{14 C}$ & $\mathbf{U}_{21 \mathrm{C}}$ & $\mathbf{U}_{7 \mathrm{~K}}$ & $\mathrm{U}_{14 \mathrm{~K}}$ & $\mathbf{U}_{21 \mathrm{~K}}$ \\
\hline A1 & Hexanal (44) & $\begin{array}{c}(140.5 \pm \\
4.6)^{\mathrm{a}}\end{array}$ & $\begin{array}{l}(51.4 \pm \\
0.2)^{\mathrm{b}}\end{array}$ & n.d. ${ }^{c}$ & n.d. ${ }^{\mathrm{c}}$ & $\begin{array}{c}(52.5 \pm \\
0.8)^{\mathrm{b}}\end{array}$ & n.d. ${ }^{\mathrm{c}}$ & n.d. ${ }^{\mathrm{c}}$ \\
\hline $\mathbf{A 2}$ & $\begin{array}{c}\text { 1-Propanol 2- } \\
\text { methyl (43) }\end{array}$ & $\underset{c}{\text { n.d. }}$ & $\underset{c}{\text { n.d. }}$ & $\begin{array}{c}(17.54 \pm \\
0.02)^{\mathrm{a}} \\
\end{array}$ & $\begin{array}{c}(18.1 \pm \\
0.2)^{\mathrm{a}} \\
\end{array}$ & n.d. ${ }^{c}$ & $\begin{array}{c}(12.2 \pm \\
1.4)^{\mathrm{b}} \\
\end{array}$ & $\begin{array}{l}(13.8 \pm \\
0.08)^{\mathrm{b}}\end{array}$ \\
\hline A3 & Pyridine (79) & n.d. ${ }^{\mathbf{b}}$ & n.d. ${ }^{b}$ & n.d. ${ }^{b}$ & n.d. & n.d. ${ }^{b}$ & n. $\mathrm{d}^{\mathrm{b} .}$ & $\begin{array}{l}(7.1 \pm \\
0.3)^{\mathrm{a}}\end{array}$ \\
\hline A4 & $\begin{array}{l}\text { 1-Butanol 2- } \\
\text { methyl (57) }\end{array}$ & $\begin{array}{l}(1.9 \pm \\
0.05)^{\mathrm{c}}\end{array}$ & $\begin{array}{l}(2.9 \pm \\
1.2)^{\mathrm{bc}}\end{array}$ & $\begin{array}{c}(11.69 \pm \\
0.14)^{\mathrm{a}}\end{array}$ & $(5.0 \pm 0.7)^{\mathrm{b}}$ & $\begin{array}{l}(3.1 \pm \\
0.2)^{\mathrm{bc}}\end{array}$ & $\begin{array}{l}(1.23 \pm \\
0.08)^{\mathrm{c}}\end{array}$ & $\begin{array}{l}(2.12 \pm \\
0.07)^{\mathrm{bc}}\end{array}$ \\
\hline A5 & $\begin{array}{l}\text { 1-Butanol 3- } \\
\text { methyl (70) }\end{array}$ & $\begin{array}{l}(2.09 \pm \\
0.04)^{\mathrm{d}}\end{array}$ & $\begin{array}{l}(3.5 \pm \\
0.4)^{\mathrm{d}} \\
\end{array}$ & $\begin{array}{c}(12.8 \pm \\
0.6)^{\mathrm{a}} \\
\end{array}$ & $\begin{array}{l}(5.5 \pm \\
0.3)^{\mathrm{c}} \\
\end{array}$ & $\begin{array}{l}(3.2 \pm \\
0.2)^{\mathrm{d}}\end{array}$ & $\begin{array}{l}(9.3 \pm \\
0.6)^{\mathrm{b}}\end{array}$ & $\begin{array}{l}(2.21 \pm \\
0.02)^{\mathrm{d}}\end{array}$ \\
\hline A6 & Limonene (68) & $\begin{array}{l}(0.82 \pm \\
0.01)^{\mathrm{b}} \\
\end{array}$ & \begin{tabular}{|l}
$(2.42 \pm$ \\
$0.06)^{\mathrm{a}}$ \\
\end{tabular} & $\begin{array}{l}(1.02 \pm \\
0.02)^{\mathrm{b}} \\
\end{array}$ & $\begin{array}{l}(1.14 \pm \\
0.04)^{\mathrm{b}} \\
\end{array}$ & $\begin{array}{l}(3.00 \pm \\
0.02)^{\mathrm{a}} \\
\end{array}$ & $\begin{array}{l}(1.10 \pm \\
0.14)^{\mathrm{b}} \\
\end{array}$ & $\begin{array}{l}(1.83 \pm \\
0.02)^{\mathrm{ab}}\end{array}$ \\
\hline A7 & $\begin{array}{c}\text { 2-Hexenal (E) } \\
(41)\end{array}$ & $(146 \pm 3)^{\mathrm{a}}$ & $\begin{array}{l}(28.3 \pm \\
0.9)^{\mathrm{b}} \\
\end{array}$ & $\begin{array}{c}(2.0 \\
\pm 1.1)^{\mathrm{c}} \\
\end{array}$ & $\begin{array}{c}(1.00 \\
\pm 0.00)^{\mathrm{c}}\end{array}$ & $(37 \pm 6)^{b}$ & n.d. ${ }^{\mathrm{c}}$ & n.d. ${ }^{c}$ \\
\hline A8 & $\begin{array}{l}\text { Cyclodecane } \\
\text { methyl (55) }\end{array}$ & $\begin{array}{c}(16.3 \pm \\
0.3)^{\mathrm{a}} \\
\end{array}$ & $\begin{array}{c}(15.1 \pm \\
0.4)^{\mathrm{a}}\end{array}$ & $\begin{array}{c}(10.34 \pm \\
0.14)^{\mathrm{c}}\end{array}$ & $\begin{array}{c}(10.9 \pm \\
1.1)^{\mathrm{c}}\end{array}$ & $\begin{array}{l}(11.2 \pm \\
0.2)^{\mathrm{bc}}\end{array}$ & $\begin{array}{c}(13.1 \pm \\
0.3)^{\mathrm{b}}\end{array}$ & $\begin{array}{l}(11.3 \pm \\
0.2)^{\mathrm{bc}}\end{array}$ \\
\hline A9 & $\begin{array}{c}\text { 2-Butanone 3- } \\
\text { hydroxy (45) }\end{array}$ & $\begin{array}{l}(4.02 \pm \\
0.11)^{\mathrm{d}} \\
\end{array}$ & $\begin{array}{l}(9.6 \pm \\
0.5)^{\mathrm{cd}} \\
\end{array}$ & $\begin{array}{l}(12.1 \pm \\
0.2)^{\mathrm{bcc}} \\
\end{array}$ & $\begin{array}{l}(17.37 \\
\pm 0.08)^{\mathrm{b}} \\
\end{array}$ & $\begin{array}{l}(9.0 \pm \\
0.7)^{\mathrm{cd}}\end{array}$ & $(39 \pm 4)^{\mathrm{a}}$ & $\begin{array}{l}(8.6 \pm \\
0.4)^{\mathrm{cd}}\end{array}$ \\
\hline A10 & Tridecane (57) & $\begin{array}{c}3.53 \\
\pm 0.01)^{\mathrm{a}} \\
\end{array}$ & $(4 \pm 2)^{\mathrm{a}}$ & $\begin{array}{l}(2.44 \pm \\
0.08)^{\mathrm{a}} \\
\end{array}$ & $\begin{array}{c}(2.3 \pm \\
0.3)^{\mathrm{a}} \\
\end{array}$ & $\begin{array}{l}(2.02 \pm \\
0.08)^{\mathrm{a}} \\
\end{array}$ & $\begin{array}{c}(3.5 \pm \\
0.8)^{\mathrm{a}} \\
\end{array}$ & $\begin{array}{l}(2.32 \pm \\
0.04)^{\mathrm{a}} \\
\end{array}$ \\
\hline A11 & $\begin{array}{c}\text { 5-Hepten-2-one. } \\
\text { 6-methyl (43) }\end{array}$ & $\begin{array}{l}(1.64 \pm \\
0.04)^{\mathrm{de}}\end{array}$ & $\begin{array}{l}(1.4 \pm \\
0.5)^{\mathrm{e}}\end{array}$ & $\begin{array}{l}(3.0 \pm \\
0.3)^{\mathrm{c}}\end{array}$ & $\begin{array}{l}(3.32 \pm \\
0.09)^{\mathrm{bc}}\end{array}$ & $\begin{array}{l}(2.6 \pm \\
0.2)^{\mathrm{cd}}\end{array}$ & $\begin{array}{l}(4.52 \pm \\
0.12)^{\mathrm{a}}\end{array}$ & $\begin{array}{l}(4.02 \pm \\
0.06)^{\mathrm{ab}}\end{array}$ \\
\hline A12 & 1-Hexanol (56) & $\begin{array}{c}(46.8 \pm \\
0.9)^{\mathrm{d}} \\
\end{array}$ & $\begin{array}{c}(186 \pm \\
2)^{\mathrm{a}} \\
\end{array}$ & $\begin{array}{c}(91.9 \pm \\
1.2)^{\mathrm{c}} \\
\end{array}$ & $\begin{array}{c}(51.3 \pm \\
0.2)^{\mathrm{d}} \\
\end{array}$ & $\begin{array}{c}(114 \pm \\
2)^{\mathrm{b}} \\
\end{array}$ & $\begin{array}{c}(18.7 \pm \\
0.2)^{\mathrm{f}} \\
\end{array}$ & $\begin{array}{c}(34.3 \pm \\
0.3)^{\mathrm{e}}\end{array}$ \\
\hline A13 & $\begin{array}{c}\text { 3-Hexen-1-ol (Z) } \\
(41)\end{array}$ & $\begin{array}{l}(5.00 \pm \\
0.09)^{\mathrm{a}} \\
\end{array}$ & $\begin{array}{l}(4.3 \pm \\
0.3)^{\mathrm{ab}} \\
\end{array}$ & $\begin{array}{c}(2.5 \pm \\
0.8)^{\mathrm{bcd}} \\
\end{array}$ & $\begin{array}{l}(2.0 \pm \\
0.4)^{\mathrm{cd}}\end{array}$ & $\begin{array}{c}(3.8 \pm \\
0.2)^{\mathrm{abc}}\end{array}$ & n.d. ${ }^{\mathrm{e}}$ & $\begin{array}{l}(1.2 \pm \\
0.2)^{\mathrm{de}}\end{array}$ \\
\hline A14 & Nonanal (57) & $(6.2 \pm 0.2)^{\mathrm{a}}$ & $(3 \pm 2)^{b}$ & $\begin{array}{l}(2.8 \pm \\
0.6)^{\mathrm{b}}\end{array}$ & $\begin{array}{c}(1.3 \pm \\
0.5)^{\mathrm{b}}\end{array}$ & $\begin{array}{l}(1.65 \pm \\
0.12)^{b}\end{array}$ & $\begin{array}{l}(1.9 \pm \\
0.5)^{\mathrm{b}}\end{array}$ & $\begin{array}{l}(1.75 \pm \\
0.09)^{\mathrm{b}}\end{array}$ \\
\hline A15 & $\begin{array}{c}\text { 2-Hexen-1-ol (E) } \\
(57)\end{array}$ & $\begin{array}{c}(30.8 \pm \\
0.2)^{\mathrm{a}}\end{array}$ & $\begin{array}{c}(128 \pm \\
2)^{\mathrm{a}}\end{array}$ & $\begin{array}{c}(45.1 \pm \\
0.8)^{\mathrm{b}}\end{array}$ & $\begin{array}{c}(23.95 \pm \\
0.11)^{\mathrm{c}}\end{array}$ & $(71 \pm 3)^{\mathrm{a}}$ & $\begin{array}{c}(1.1 \pm \\
0.3)^{\mathrm{a}} \\
\end{array}$ & $\begin{array}{c}(22.6 \pm \\
0.8)^{\mathrm{a}}\end{array}$ \\
\hline A16 & Acetic acid (45) & $\begin{array}{c}(21.8 \pm \\
0.5)^{\mathrm{a}}\end{array}$ & $\begin{array}{c}(19 \pm \\
2)^{\mathrm{a}}\end{array}$ & $\begin{array}{c}(11.6 \pm \\
0.8)^{\mathrm{a}}\end{array}$ & $\begin{array}{c}(16.4 \pm \\
0.4)^{\mathrm{a}}\end{array}$ & $\begin{array}{c}(13.3 \pm \\
0.9)^{\mathrm{a}}\end{array}$ & $\begin{array}{c}(15.01 \pm \\
0.08)^{\mathrm{a}}\end{array}$ & $\begin{array}{c}(13 \pm \\
8)^{\mathrm{a}}\end{array}$ \\
\hline A17 & $\begin{array}{c}\text { Cycloisosativene } \\
(147)\end{array}$ & $\begin{array}{l}(1.27 \pm \\
0.04)^{\mathrm{a}} \\
\end{array}$ & $\begin{array}{l}(1.81 \pm \\
0.04)^{\mathrm{a}} \\
\end{array}$ & $\begin{array}{l}(2.66 \pm \\
0.05)^{\mathrm{a}} \\
\end{array}$ & $\begin{array}{l}(2.82 \pm \\
0.05)^{\mathrm{a}} \\
\end{array}$ & $\begin{array}{l}(1.66 \pm \\
0.14)^{\mathrm{a}} \\
\end{array}$ & $\begin{array}{c}(3.0 \pm \\
0.3)^{\mathrm{a}} \\
\end{array}$ & $\begin{array}{l}(2.8 \pm \\
0.2)^{\mathrm{a}} \\
\end{array}$ \\
\hline A18 & n.i. (119) & $\begin{array}{l}(2.32 \pm \\
0.07)^{\mathrm{a}} \\
\end{array}$ & $\begin{array}{c}(2.4 \pm \\
0.2)^{\mathrm{a}} \\
\end{array}$ & $\begin{array}{l}(1.49 \pm \\
0.08)^{\mathrm{b}}\end{array}$ & $\begin{array}{l}(1.69 \pm \\
0.05)^{\mathrm{b}}\end{array}$ & $\begin{array}{l}(1.66 \pm \\
0.01)^{\mathrm{b}}\end{array}$ & $\begin{array}{l}(1.9 \pm \\
0.2)^{\mathrm{b}}\end{array}$ & $\begin{array}{l}(1.7 \pm \\
0.1)^{\mathrm{b}}\end{array}$ \\
\hline A19 & $\begin{array}{c}\text { Dimethyl } \\
\text { sulfoxide (63) }\end{array}$ & $\begin{array}{c}(13.3 \pm \\
0.3)^{\mathrm{b}}\end{array}$ & $\begin{array}{l}(2.0 \pm \\
0.3)^{\mathrm{d}} \\
\end{array}$ & $\begin{array}{c}(14.7 \pm \\
0.2)^{\mathrm{a}} \\
\end{array}$ & $\begin{array}{l}(14.0 \pm \\
0.4)^{\mathrm{ab}}\end{array}$ & $\begin{array}{l}(2.1 \pm \\
0.3)^{\mathrm{d}} \\
\end{array}$ & $\begin{array}{l}(2.2 \pm \\
0.5)^{d}\end{array}$ & $\begin{array}{l}(4.75 \pm \\
0.13)^{\mathrm{c}}\end{array}$ \\
\hline $\mathbf{A 2 0}$ & $\begin{array}{c}\alpha \text {-Bergamotene } \\
\text { (119) }\end{array}$ & $\begin{array}{l}(2.32 \pm \\
0.01)^{\mathrm{a}}\end{array}$ & $\begin{array}{l}(2.35 \pm \\
0.03)^{\mathrm{a}}\end{array}$ & $\begin{array}{l}(1.62 \pm \\
0.07)^{\mathrm{bc}}\end{array}$ & $\begin{array}{l}(1.7 \pm \\
0.2)^{\mathrm{bc}}\end{array}$ & $\begin{array}{l}(1.42 \pm \\
0.13)^{\mathrm{c}}\end{array}$ & $\begin{array}{l}(1.96 \pm \\
0.01)^{\mathrm{ab}}\end{array}$ & $\begin{array}{l}(1.7 \pm \\
0.2)^{\mathrm{bc}}\end{array}$ \\
\hline
\end{tabular}




\begin{tabular}{|c|c|c|c|c|c|c|c|c|}
\hline & & & & & & & able 4* ( & continue) \\
\hline & & & Norn & nalised pe & ak area $(\mathrm{m}$ & $\operatorname{ean} \pm$ SD & $n=2)$ & \\
\hline$\overline{0}$ & Compounds & & & $\mathbf{U}_{\mathbf{C}}$ & & & $\mathbf{U}_{\mathbf{K}}$ & \\
\hline & $(\mathbf{m} / \mathbf{z})$ & $\mathbf{U}_{0}$ & $\mathbf{U}_{7 \mathrm{C}}$ & $\mathbf{U}_{14 \mathrm{C}}$ & $\mathbf{U}_{21 \mathrm{C}}$ & $\mathbf{U}_{7 \mathrm{~K}}$ & $\mathrm{U}_{14 \mathrm{~K}}$ & $\mathrm{U}_{21 \mathrm{~K}}$ \\
\hline A21 & $\begin{array}{c}\text { Decanoic acid } \\
\text { methyl ester (74) }\end{array}$ & n.d. ${ }^{a}$ & n.d. ${ }^{a}$ & n.d. ${ }^{a}$ & n.d. ${ }^{a}$ & n.d. ${ }^{a}$ & n.d. ${ }^{a}$ & n.d. ${ }^{a}$ \\
\hline A22 & $\begin{array}{l}\text { Cycloheptanone } \\
\text { 2-methylene (43) }\end{array}$ & n.d. ${ }^{a}$ & n.d. ${ }^{a}$ & n.d. ${ }^{a}$ & n.d. ${ }^{a}$ & n.d. ${ }^{a}$ & n.d. ${ }^{a}$ & n.d. ${ }^{a}$ \\
\hline $\mathbf{A 2 3}$ & $\begin{array}{c}\text { (E)- } \beta \text {-Farnesene } \\
(69)\end{array}$ & $\begin{array}{l}(6.70 \pm \\
0.18)^{\mathrm{ab}}\end{array}$ & $\begin{array}{l}(7.1 \pm \\
0.7)^{\mathrm{a}} \\
\end{array}$ & $\begin{array}{l}(3.8 \pm \\
0.3)^{\mathrm{cd}}\end{array}$ & $\begin{array}{l}(4.8 \pm \\
0.5)^{\mathrm{bcd}}\end{array}$ & $\begin{array}{l}(3.27 \pm \\
0.14)^{\mathrm{d}} \\
\end{array}$ & $\begin{array}{l}5.5 \pm \\
0.2)^{\mathrm{abc}}\end{array}$ & $\begin{array}{l}(4.7 \pm \\
0.9)^{\mathrm{cd}}\end{array}$ \\
\hline A24 & $\begin{array}{c}\text { 8-Heptadecene } \\
(69)\end{array}$ & $\begin{array}{l}(7.64 \pm \\
0.10)^{\mathrm{a}} \\
\end{array}$ & $(7 \pm 2)^{\mathrm{a}}$ & $\begin{array}{l}(2.7 \pm \\
0.4)^{\mathrm{b}}\end{array}$ & $\begin{array}{c}(3.1 \pm \\
0.5)^{\mathrm{b}} \\
\end{array}$ & $\begin{array}{l}(2.7 \pm \\
0.3)^{\mathrm{b}}\end{array}$ & $\begin{array}{l}(3.6 \pm \\
0.3)^{\mathrm{b}}\end{array}$ & $\begin{array}{l}(3.7 \pm \\
0.6)^{\mathrm{b}}\end{array}$ \\
\hline A25 & $\begin{array}{c}\text { Eremophilene } \\
(161)\end{array}$ & $\begin{array}{c}(10.7 \pm \\
0.4)^{\mathrm{a}} \\
\end{array}$ & $\begin{array}{l}(13.8 \pm \\
1.2)^{\mathrm{b}} \\
\end{array}$ & \begin{tabular}{|c|}
$(12.3 \pm$ \\
$0.4)^{\mathrm{bc}}$ \\
\end{tabular} & $\begin{array}{l}(11.9 \pm \\
1.0)^{\mathrm{bc}} \\
\end{array}$ & $\begin{array}{l}(9.6 \pm \\
1.0)^{\mathrm{c}}\end{array}$ & $\begin{array}{c}(17.7 \pm \\
0.3)^{\mathrm{a}} \\
\end{array}$ & $\begin{array}{l}(11.0 \pm \\
1.3)^{\mathrm{bc}} \\
\end{array}$ \\
\hline A26 & $\alpha$-Farnesene (93) & $(614 \pm 2)^{\mathrm{a}}$ & $\begin{array}{c}(570 \pm \\
90)^{\mathrm{a}} \\
\end{array}$ & $\begin{array}{c}(219.70 \pm \\
0.05)^{\mathrm{b}} \\
\end{array}$ & $\begin{array}{c}(230 \pm \\
30)^{\mathrm{b}} \\
\end{array}$ & $\begin{array}{c}(200 \pm \\
20)^{\mathrm{b}}\end{array}$ & $\begin{array}{c}(259 \pm \\
6)^{\mathrm{b}} \\
\end{array}$ & $\begin{array}{c}(210 \pm \\
20)^{\mathrm{b}} \\
\end{array}$ \\
\hline A27 & $\begin{array}{c}\beta- \\
\text { Sesquiphellandre } \\
\text { ne (69) }\end{array}$ & n.d. ${ }^{a}$ & n.d. ${ }^{a}$ & n.d. ${ }^{a}$ & n.d. ${ }^{a}$ & n.d. ${ }^{a}$ & n.d. ${ }^{a}$ & n.d. ${ }^{a}$ \\
\hline A28 & $\begin{array}{l}\text { Dodecanoic acid. } \\
\text { methyl ester (74) }\end{array}$ & $\begin{array}{l}(1.5 \pm \\
0.01)^{\mathrm{a}}\end{array}$ & $\begin{array}{l}(0.7 \pm \\
0.2)^{\mathrm{ab}} \\
\end{array}$ & n.d. ${ }^{b}$ & n.d. ${ }^{b}$ & $\begin{array}{l}(0.91 \pm \\
0.13)^{\mathrm{ab}}\end{array}$ & $\begin{array}{l}(1.11 \pm \\
0.13)^{\mathrm{a}} \\
\end{array}$ & n.d. ${ }^{\mathrm{b}}$ \\
\hline A29 & $\begin{array}{c}\text { Hexanoic acid } \\
(60)\end{array}$ & $\begin{array}{c}(20.4 \pm \\
0.4)^{\mathrm{a}}\end{array}$ & $\begin{array}{l}(15.4 \pm \\
1.3)^{\mathrm{b}} \\
\end{array}$ & $(8 \pm 2)^{\mathrm{cd}}$ & $\begin{array}{l}(3.4 \pm \\
0.7)^{\mathrm{ef}}\end{array}$ & $\begin{array}{l}(9.9 \pm \\
0.5)^{\mathrm{c}}\end{array}$ & $\begin{array}{l}(4.1 \pm \\
0.2)^{\mathrm{de}}\end{array}$ & n.d. ${ }^{f}$ \\
\hline $\mathbf{A 3 0}$ & $\begin{array}{l}\text { Phenylethyl } \\
\text { alcohol (91) }\end{array}$ & $\begin{array}{l}(6.20 \pm \\
0.12)^{b} \\
\end{array}$ & \begin{tabular}{|l|}
$(6.7 \pm$ \\
$0.3)^{\mathrm{b}}$ \\
\end{tabular} & $\begin{array}{r}(80 \pm \\
30)^{\mathrm{a}} \\
\end{array}$ & $\begin{array}{l}(28.6 \pm \\
0.6)^{\mathrm{b}}\end{array}$ & $\begin{array}{l}(6.9 \pm \\
0.2)^{\mathrm{b}} \\
\end{array}$ & $\begin{array}{c}(23.4 \pm \\
0.8)^{\mathrm{b}}\end{array}$ & $\begin{array}{l}(18.2 \pm \\
0.8)^{\mathrm{b}} \\
\end{array}$ \\
\hline A31 & $\begin{array}{c}\text { 2.6-Bis(1.1- } \\
\text { dimethylethyl)-4- } \\
\text { (1-oxopropyl) } \\
\text { phenol (233) }\end{array}$ & $\begin{array}{l}(9.70 \pm \\
0.13)^{\mathrm{ab}}\end{array}$ & $\begin{array}{c}(10.6 \pm \\
0.7)^{\mathrm{a}}\end{array}$ & $\begin{array}{c}(10.06 \pm \\
0.04)^{\mathrm{ab}}\end{array}$ & $\begin{array}{l}(9.10 \pm \\
0.11)^{b}\end{array}$ & $\begin{array}{l}(9.4 \pm \\
0.4)^{\mathrm{ab}}\end{array}$ & $\begin{array}{c}(10.3 \pm \\
0.5)^{\mathrm{ab}}\end{array}$ & $\begin{array}{l}(8.95 \pm \\
0.070^{b}\end{array}$ \\
\hline A32 & $\begin{array}{l}\text { 4.6-Heptadienoic } \\
\text { acid 3.3.6- } \\
\text { trimethyl. methyl } \\
\text { ester (109) }\end{array}$ & n.d. ${ }^{b}$ & $(5 \pm 3)^{\mathrm{ab}}$ & $\begin{array}{l}(7.8 \pm \\
1.4)^{\mathrm{a}}\end{array}$ & $\begin{array}{l}(1.4 \pm \\
0.6)^{b}\end{array}$ & n.d. ${ }^{b}$ & $\begin{array}{l}(0.94 \pm \\
0.08)^{\mathrm{b}}\end{array}$ & $\begin{array}{l}(1.4 \pm \\
0.5)^{\mathrm{b}}\end{array}$ \\
\hline $\mathbf{A 3 3}$ & n.i. (159) & $\begin{array}{l}(7.72 \pm \\
0.13)^{\mathrm{a}} \\
\end{array}$ & $(3 \pm 2)^{b}$ & $\begin{array}{l}(4.54 \pm \\
0.10)^{\mathrm{ab}}\end{array}$ & $\begin{array}{l}(2.4 \pm \\
0.5)^{\mathrm{ab}} \\
\end{array}$ & $\begin{array}{l}(1.4 \pm \\
0.6)^{\mathrm{b}} \\
\end{array}$ & $(2 \pm 2)^{b}$ & $\begin{array}{l}(2.3 \pm \\
0.2)^{\mathrm{ab}} \\
\end{array}$ \\
\hline A34 & $\begin{array}{c}\text { 3-Buten-2-ol. 2- } \\
\text { methyl (71) }\end{array}$ & $\begin{array}{l}(3.31 \pm \\
0.10)^{\mathrm{a}} \\
\end{array}$ & n.d. ${ }^{b}$ & n.d. ${ }^{b}$ & $\mathrm{n} \cdot \mathrm{d}^{\mathrm{b}}$ & n.d. ${ }^{b}$ & n.d. ${ }^{b}$ & n.d. ${ }^{b}$ \\
\hline A35 & 1-Dodecanol (55) & $\begin{array}{c}\text { n.d. } \\
\text { a }\end{array}$ & $\begin{array}{c}\text { n.d. } \\
\text { a }\end{array}$ & $\begin{array}{c}\text { n.d. } \\
\text { a }\end{array}$ & $\begin{array}{c}\text { n.d. } \\
\text { a }\end{array}$ & $\begin{array}{c}\text { n.d. } \\
\text { a }\end{array}$ & $\begin{array}{c}\text { n.d. } \\
\text { a }\end{array}$ & $\begin{array}{c}\text { n.d. } \\
\text { a }\end{array}$ \\
\hline A36 & $\begin{array}{l}\text { Dimethylpalmita } \\
\text { mine (58) }\end{array}$ & $\begin{array}{l}(8.2 \pm \\
0.2)^{\mathrm{a}} \\
\end{array}$ & $\begin{array}{l}(8.1 \pm \\
0.6)^{\mathrm{a}} \\
\end{array}$ & $(16 \pm 5)^{\mathrm{a}}$ & $(12 \pm 6)^{\mathrm{a}}$ & $\begin{array}{l}(4.0 \pm \\
0.4)^{\mathrm{a}} \\
\end{array}$ & $\begin{array}{l}(6.8 \pm \\
0.9)^{\mathrm{a}} \\
\end{array}$ & $\begin{array}{l}(7.3 \pm \\
0.7)^{\mathrm{a}} \\
\end{array}$ \\
\hline $\mathbf{A 3 7}$ & $\begin{array}{c}\text { Methyl } \\
\text { tetradecanoate } \\
(74) \\
\end{array}$ & n.d. ${ }^{a}$ & n.d. ${ }^{a}$ & n.d. ${ }^{a}$ & n.d. ${ }^{a}$ & n.d. ${ }^{a}$ & n.d. ${ }^{a}$ & n.d. ${ }^{\mathrm{a}}$ \\
\hline A38 & Phenol (94) & $\begin{array}{l}(3.43 \pm \\
0.07)^{\mathrm{b}} \\
\end{array}$ & $\begin{array}{l}(2.2 \pm \\
0.4)^{\mathrm{c}} \\
\end{array}$ & $(9 \pm 2)^{\mathrm{a}}$ & $\begin{array}{l}(6.18 \pm \\
0.09)^{b} \\
\end{array}$ & $\begin{array}{l}(2.0 \pm \\
0.5)^{\mathrm{c}} \\
\end{array}$ & $\begin{array}{l}(3.43 \pm \\
0.08)^{\mathrm{bc}}\end{array}$ & $\begin{array}{l}3.08 \pm \\
0.09)^{\mathrm{bc}} \\
\end{array}$ \\
\hline A39 & Nerolidol 2 (69) & $(54 \pm 2)^{\mathrm{a}}$ & $(21 \pm 10)^{b}$ & $(15 \pm 2)^{b c}$ & $(3.3 \pm 0.8)^{\mathrm{c}}$ & $\begin{array}{l}(3.0 \pm \\
1.4)^{\mathrm{c}}\end{array}$ & $\begin{array}{l}(7.0 \pm \\
0.2)^{\mathrm{bc}}\end{array}$ & $\begin{array}{l}(2.59 \pm \\
0.04)^{\mathrm{c}} \\
\end{array}$ \\
\hline A40 & $\begin{array}{c}\text { 2(3H)-Furanone } \\
\text { dihydro-5-propyl } \\
(85)\end{array}$ & $\begin{array}{l}(8.07 \pm \\
0.12)^{b}\end{array}$ & $(9 \pm 2)^{b}$ & $(25 \pm 9)^{\mathrm{a}}$ & $\begin{array}{l}(5.9 \pm \\
0.7)^{\mathrm{b}}\end{array}$ & $\begin{array}{l}(4.6 \pm \\
0.3)^{\mathrm{b}}\end{array}$ & $\begin{array}{l}(12.44 \pm \\
0.04)^{\mathrm{ab}}\end{array}$ & $\begin{array}{l}(4.21 \pm \\
0.04)^{\mathrm{b}}\end{array}$ \\
\hline
\end{tabular}




\begin{tabular}{|c|c|c|c|c|c|c|c|c|}
\hline \multicolumn{9}{|c|}{ Table 4* (continue) } \\
\hline \multirow{3}{*}{$\frac{\mathscr{g}}{8}$} & \multirow{3}{*}{$\begin{array}{c}\text { Compounds } \\
(\mathbf{m} / \mathbf{z})\end{array}$} & \multicolumn{7}{|c|}{ Normalised peak area $($ mean \pm SD. $n=2)$} \\
\hline & & & \multicolumn{3}{|c|}{$\mathbf{U}_{\mathbf{C}}$} & \multicolumn{3}{|c|}{$\mathbf{U}_{\mathbf{K}}$} \\
\hline & & $\mathbf{U}_{\mathbf{0}}$ & $\mathbf{U}_{7 \mathrm{C}}$ & $\mathrm{U}_{14 \mathrm{C}}$ & $\mathbf{U}_{21 C}$ & $\mathbf{U}_{7 \mathbf{K}}$ & $\mathrm{U}_{14 \mathrm{~K}}$ & $\mathbf{U}_{21 \mathrm{~K}}$ \\
\hline A41 & $\begin{array}{l}\text { Benzoic acid. 2- } \\
\text { methoxy. methyl } \\
\text { ester (135) }\end{array}$ & $\begin{array}{l}(18.0 \\
\pm 0.5)^{\mathrm{a}}\end{array}$ & $\begin{array}{l}(7.4 \pm \\
0.5)^{b}\end{array}$ & $\begin{array}{l}(3.7 \pm \\
1.3)^{\mathrm{c}}\end{array}$ & $(1.2 \pm 0.2)^{\mathrm{d}}$ & $\begin{array}{l}(2.79 \pm \\
0.04)^{\mathrm{cd}}\end{array}$ & $\begin{array}{l}(2.73 \pm \\
0.02)^{\mathrm{cd}}\end{array}$ & $\begin{array}{l}(1.7 \pm \\
0.2)^{\mathrm{cd}}\end{array}$ \\
\hline A42 & $\begin{array}{l}\text { 2H-Pyran-2-one. } \\
6- \\
\text { hexyltetrahydro } \\
\text { (99) }\end{array}$ & $\begin{array}{c}(14.83 \\
\pm 0.50)^{\mathrm{a}}\end{array}$ & $\begin{array}{c}(11 \\
\pm 5)^{\mathrm{ab}}\end{array}$ & $\begin{array}{c}(10.8 \\
\pm 0.8)^{\mathrm{ab}}\end{array}$ & $\begin{array}{c}(1.8 \\
\pm 0.2)^{\mathrm{c}}\end{array}$ & $\begin{array}{c}(2.1 \\
\pm 0.6)^{\mathrm{c}}\end{array}$ & $\begin{aligned} & (5.5 \\
\pm & 0.3)^{\mathrm{bc}}\end{aligned}$ & $\begin{array}{c}(1.3 \\
\pm 0.2)^{\mathrm{c}}\end{array}$ \\
\hline A43 & $\begin{array}{c}\text { Hexadecanoic } \\
\text { acid methyl ester } \\
(74)\end{array}$ & $\begin{array}{c}(5.30 \\
\pm 0.08)^{\mathrm{a}}\end{array}$ & $\begin{array}{c}(4.6 \\
\pm 0.8)^{\mathrm{a}}\end{array}$ & $\begin{array}{c}(60 \\
\pm 60)^{\mathrm{a}}\end{array}$ & $\begin{array}{c}(2.8 \\
\pm 0.4)^{\mathrm{a}}\end{array}$ & $\begin{array}{c}(2.7 \\
\pm 0.3)^{\mathrm{a}}\end{array}$ & $\begin{array}{c}(3.23 \\
\pm 0.10)^{\mathrm{a}}\end{array}$ & $\begin{array}{c}(4.1 \\
\pm 1.4)^{\mathrm{a}}\end{array}$ \\
\hline A44 & n.i. (71) & n.d. ${ }^{\mathrm{b}}$ & $\begin{array}{c}(1.4 \\
\pm 0.5)^{\mathrm{ab}}\end{array}$ & $\begin{array}{c}(5.7 \\
\pm 0.4)^{\mathrm{a}}\end{array}$ & n.d. ${ }^{b}$ & n.d. ${ }^{b}$ & $\begin{array}{c}(7.6 \\
\pm 1.2)^{\mathrm{a}} \\
\end{array}$ & n.d. ${ }^{b}$ \\
\hline A45 & $\begin{array}{l}\text { 9-Octadecenoic } \\
\text { acid methyl ester } \\
(55)\end{array}$ & $\begin{array}{c}(4.63 \pm \\
0.14)^{\mathrm{a}}\end{array}$ & $(7 \pm 4)^{\mathrm{a}}$ & $\begin{array}{c}(15 \pm \\
6)^{\mathrm{a}}\end{array}$ & $\begin{array}{l}(3.03 \pm \\
1.85)^{\mathrm{a}}\end{array}$ & $\begin{array}{l}(3.8 \pm \\
1.0)^{\mathrm{a}}\end{array}$ & $(3.3 \pm 1.0)^{\mathrm{a}}$ & $(6 \pm 2)^{\mathrm{a}}$ \\
\hline A46 & $\begin{array}{c}p- \\
\text { Isopropenylphen } \\
\text { ol (134) }\end{array}$ & $\begin{array}{l}(2.16 \pm \\
0.07)^{\mathrm{a}}\end{array}$ & $\begin{array}{l}(2.5 \pm \\
0.8)^{\mathrm{a}}\end{array}$ & $\begin{array}{l}(2.1 \pm \\
0.3)^{\mathrm{a}}\end{array}$ & $\begin{array}{l}(1.90 \pm \\
0.05)^{\mathrm{a}}\end{array}$ & $\begin{array}{c}(1.6 \pm \\
0.3)^{\mathrm{a}}\end{array}$ & $\begin{array}{c}(2.14 \pm \\
0.10)^{\mathrm{a}}\end{array}$ & $\begin{array}{l}(1.90 \pm \\
0.05)^{\mathrm{a}}\end{array}$ \\
\hline
\end{tabular}

${ }^{1}$ n.d. not detected.

Data showed mean of two independent tests (mean \pm sd).

${ }^{\mathrm{a}-\mathrm{c}}$ Values in the same row with different superscript letters differ significantly $(p<0.05)$.

The storage of olives in inappropriate conditions had a negative impact on the aroma profiles of oils. Uslu olive oil was characterized with high concentrations of terpene volatile compound such as $\alpha$-Farnesene (614 \pm 2$)$ and aldehydes as 2-hexenal (146 \pm 3$)$ and hexanal (140.5 \pm 4.6$)$. In contrast, Edremit olive oil had lower concentrations of $\alpha$-Farnesene and 2exenal, which were determined as $150 \pm 20$ and $14 \pm 1.3$, respectively. The increase of concentration of 2-hexanal (E) during holding periods could be explained by the activity of the fungal enzymes in LOX pathway of olive fruits (Schnurer et al. 1999). However, the concentration of hexanal $(251 \pm 11)$ in Edremit olive oil was immediately increased after extraction. In this study, from alcohol compounds 1-Hexanol and 3-Hexen-1-ol were found as the most potent volatiles of the Edremit oils, which concentration increased over holding time in both holding types. In Uslu olive oils, the concentration of 1-hexanol also increased significantly $(\mathrm{p}<0.05)$ during the holding time as observed for Edremit oil. The concentration of 3-Hexen-1-ol in Uslu oil during holding time did not increase in contrast to Edremit oil. Lower values of hexanal, hexanol, cis-3-hexenol and higher values of methanol, ethanol and 2-methyl butanol (fusty) in samples from plastic bags were also observed by other researchers (Koprivnjak \& Conte 2002). The presence of hexanol was in virgin olive oils also reported (Haddada et al. 2007; Krichene et al. 2010; Manai et al. 2008). However, Kaftan (2007) did not identify hexanol in Memecik olive oils of 2005 and 2006 harvesting seasons. These differences between the findings can be related to the activity of alcohol dehydrogenase enzyme. As seen from Table 3 and Table 4, phenylethyl alcohol was also detected and its content increased during holding periods in both type of olive oils. Its occurrence was due to the degradation of phenylalanine (Issaoui et al. 2009). 
Previous studies focused on relationship between irradiation or weather conditions and content of volatile compounds in olive oil before harvesting. However, no studies determined the effect of weather conditions on the content of volatile compounds in olive oil after harvesting. Servili et al. (2007) found that the presence of several volatile compounds, such as hexanal, (E)-2-hexenal, and other lipoxygenase derivative products positively correlated with the irrigation rate. In our study hexanal was not detected between 7-14 days when weather was stormy and heavy rain in both two variety of olive oils, while dimethylpalmitamine was not detected in stormy weather only in Edremit variety olive oils. The content of 2-hexen-1-ol significantly increased in Edremit olive oils holded in nylon sacks between 7-14 days. However, it was not detected in oils holded in plastic boxes. Similarly, the content of cycloheptanone 2-methylene significantly increased in Edremit olive oils holded in nylon sacks between 14-21 days (cloudy weather), however it was not detected in Uslu and Edremit oils holded in plastic boxes. While decrease in $\alpha$-Farnesene in Edremit olive oils holded in nylon sacks between 0-7 days (sunny weather) was significant, this decrease was not so great in Uslu olive oils holded in nylon sacks during the days of 0 7.

Hexanal, which is responsible for fatty, fruity, green odor perceptions and contributed to pleasant sensory perceptions, was formed due to activity of aldehyde-lyase enzyme (Table 2). Therefore, the content of hexanal has been correlated with the highest overall grading of virgin olive oil (Morales \& Aparicio 1999). In this study, the concentration of hexanal decreased more in oils obtained from Edremit variety holded at cases compared to oils extracted from Edremit variety holded at nylon sacks during 7 days. It decreased to $29.74 \pm 0.14$ and to $9 \pm 2$ in nylon sacks and case, respectively. While the ratio of hexanal to nonanal was lower than 2 , the oil was evaluated as rancid which detectes the beginning of oxidation (Kesen et al. 2013). Therefore, according to the results of $14^{\text {th }}$ and $21^{\text {th }}$ days, it has seen that hexanal did not occur in the oil because of it is under the detectable limits. This indicated that hexanal/nonanal ratio was below 2 . However, the decrease in hexanal content was not significantly different in oils obtained from Uslu variety olives which were holded for 7 days in sacks and cases ( $p>0.05$ ). Acetic acid which is responsible for pungent, sour, vinegar-like odor can be formed by microorganisms growing on or in the olives during storage and are undesirable for olive oils (Davis 2007). Because of the production of acetic acid, Acetobacter could develop and cause vinegary defect. Acetic acid concentration in this sudy was not effected significantly by holding time and holding type in both olive oils during 21 days of storage $(\mathrm{p}>0.05)$. While 5-Hepten-2-one 6-methyl and 1-Butanol 3-methyl volatiles compounds were not detected in Edremit and Uslu olive oils at initial day, they formed during both sack and box holding due to the activity of the microorganisms in olives (Table 3 and Table 4). The occurence of 5-Hepten-2-one 6-methyl can be explained by the presence of bacteria from the genus Pseudomonas which are capable to degradate terpenic alcohols (Morales 2005).

PCA was used to highlight the data and to determine the relationships between the content of volatile compounds and olive oil variety during different holding times and types (Figure 1-3). 


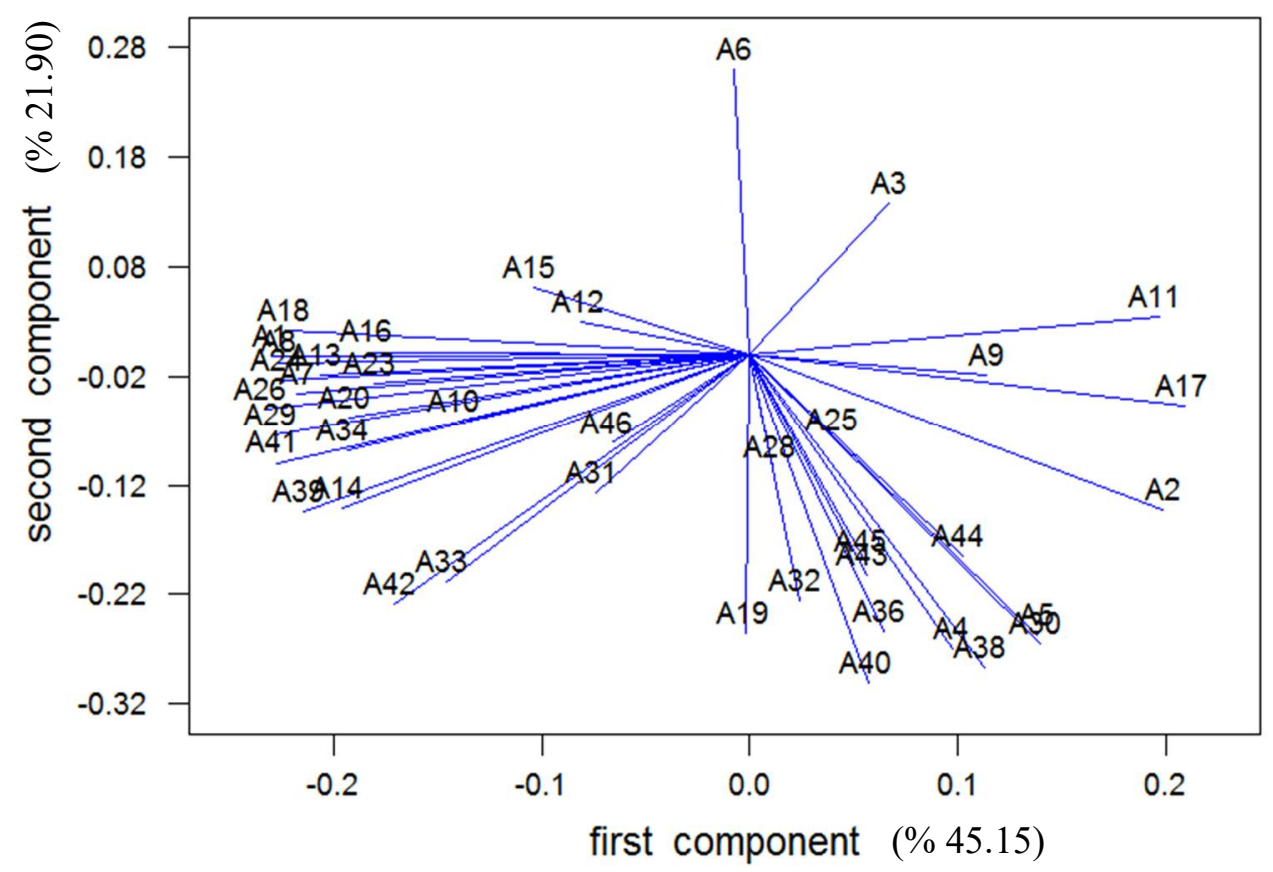

Figure 1. PCA plots of volatile compounds of Uslu variety olive oils.

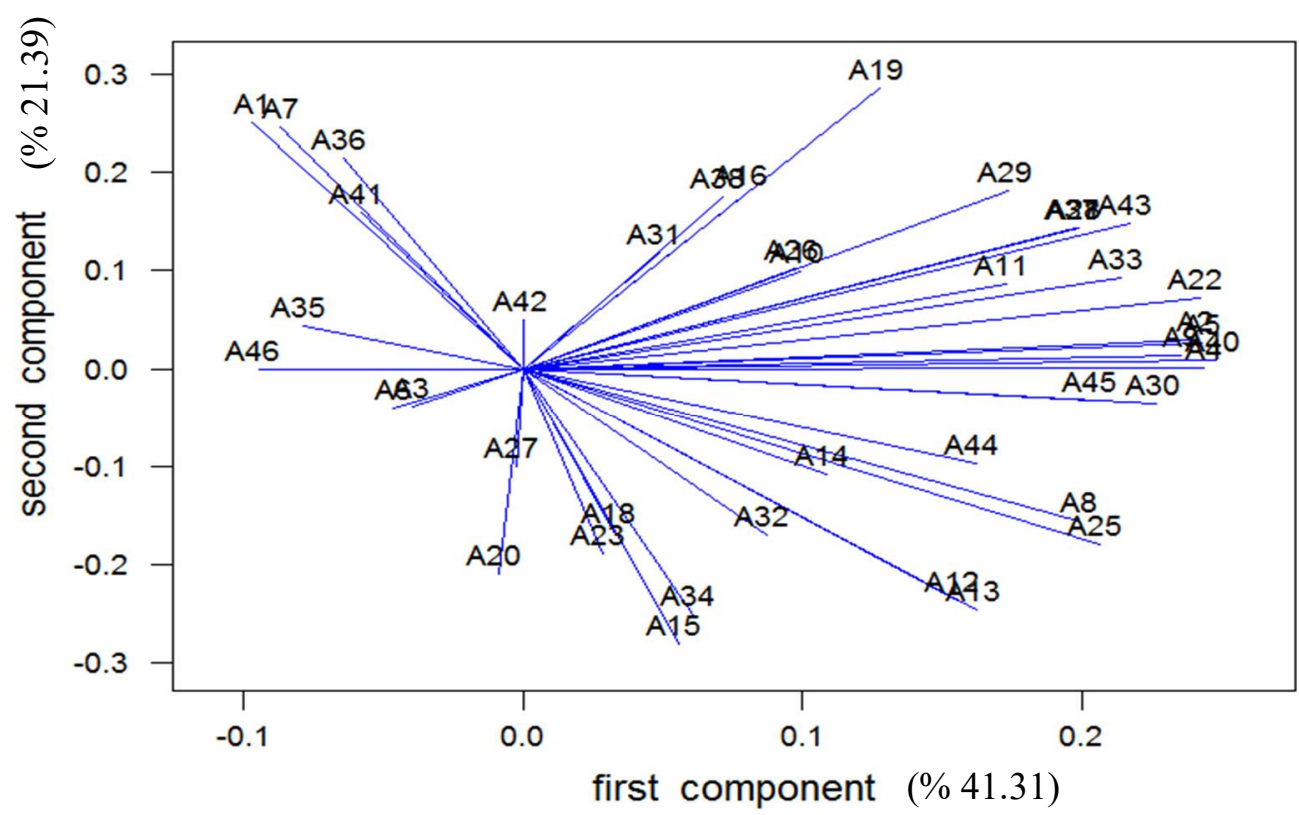

Figure 2. PCA plots of volatile compounds of Edremit variety olive oils 


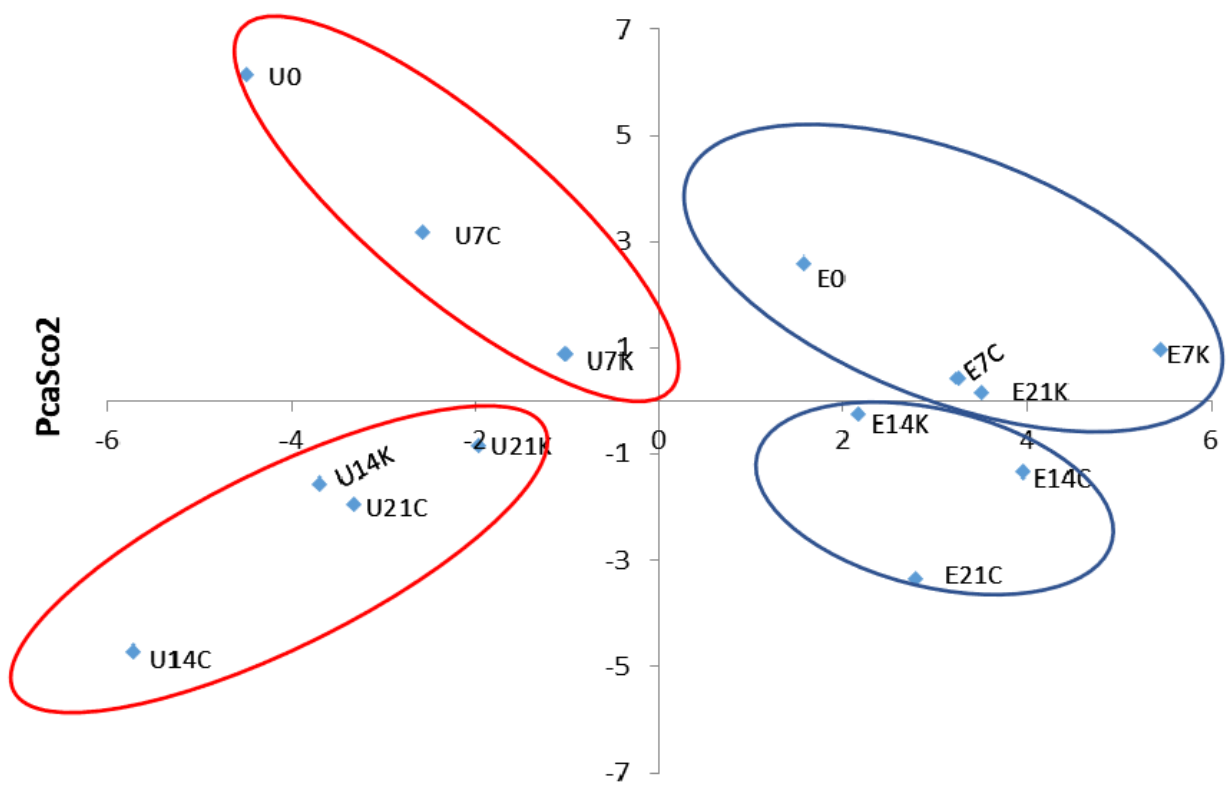

PcaSco1

Figure 3. Score plots of two first components applied to volatile compounds of olive oils extracted from Uslu and Edremit cultivars stored at case and nylon sacks until 21 days

PCA permitted a reduction of volatile compounds found in olive oil to three principal components (with eigenvalues $>1$ ). PCA results showed that 5 components were found (eigenvalues $>1$ ) for both olive varieties (Figure 4), but eigenvalues of two components were lower than the calculated percentile 95\% they were dropped and only the first 3 components which eigenvalues were greater than percentile 95\% were retained. Yang et al. (2017) used PCA and AHC chemometric analysis to determine the sensitive oxidation marker compounds, including (E, E)-2,4-heptadienal marker, by lipid oxidation in the n-3 PUFA rich oils. They found that oxidized volatiles did not rise linearly during oxidation and volatile compounds of lipid oxidation were not clearly identicable with PCA analysis (Yang et al. 2017).

Three PCs were extracted represent $81.27 \%$ of the total variance of olive oil samples extracted from Uslu cultivar and $80.14 \%$ of the total variance of olive oil samples extracted from Edremit cultivar. The first principal components PC1, PC2 and PC3 represented $45.15 \%$ and $41.31 \%, 21.90 \%$ and $21.39 \%, 14.21 \%$ and $17.43 \%$, respectively for Uslu and Edremit varieties. First component was mainly defined by A11, A17 and A2 with positive correlation and second component mainly by A42 and A33 (with a high negative correlation) in olive oils obtained from Uslu varieties (Figure 1). On the other hand, first component was mainly defined by A22, A40 and A43 and second component mainly by A19, A1 and A7 with a high positive correlation in olive oils extracted from Edremit varieties (Figure 2). 


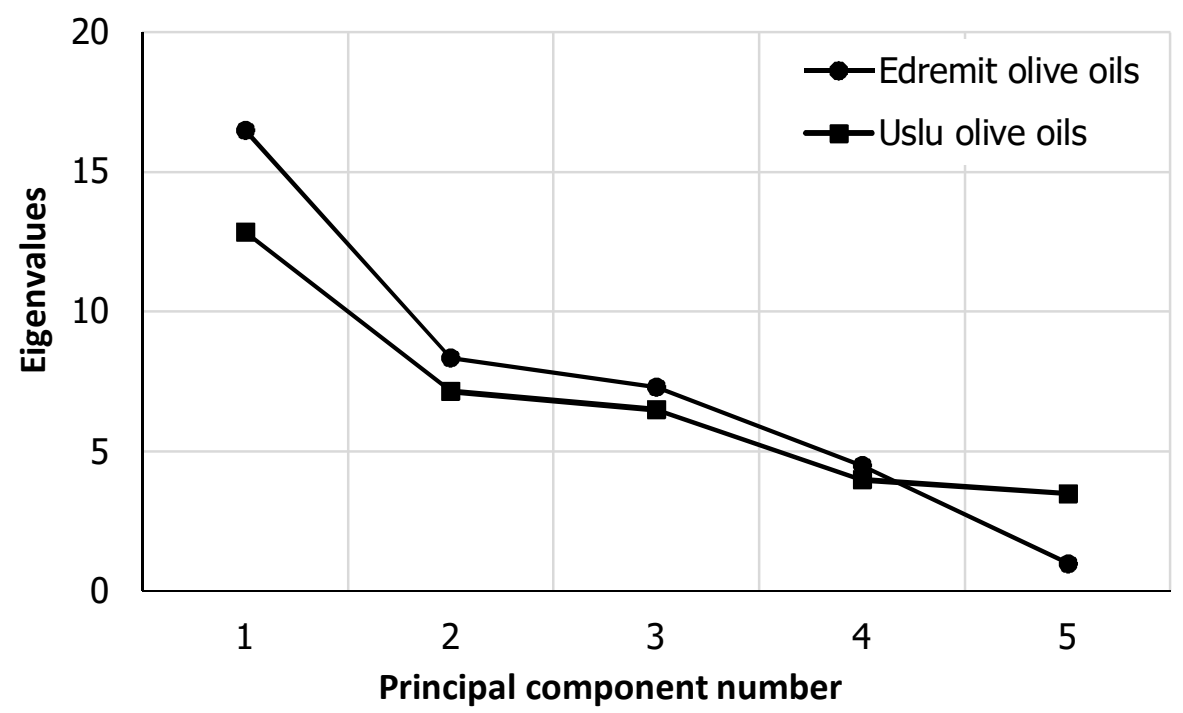

Figure 4. Eigenvalues of volatile compounds of Uslu and Edremit olive oils obtained by PCA

\section{Conclusions}

Principal component analysis (PCA) was used to evaluate the effect of different types of olive packaging (plastic box and nylon socks) and different period of olive storage in these packaging on oil aroma profiles and to find the relationships between volatile compounds and olive oil varieties. Content of hexanal decreased significantly in oils obtained from Edremit variety during 7 days of holding in case. However, the holding type had no effect on the hexanal content in Uslu olive oils. PC1, PC2 and PC3 identified the $81.27 \%$ of the total variance of olive oil samples extracted from Uslu cultivar and $80.14 \%$ of the total variance of olive oil samples extracted from Edremit cultivar. The content of acetic acid, which is an undesirable compound in virgin olive oils, was not changed significantly in both two oils depending on holding type or time.

To provide high quality of olive oils conditions for its storage should be controlled.The recommended storage conditions for olives is about $5{ }^{\circ} \mathrm{C}$ in air conditioning boxes for at least 30 days to reduce development of fungi and protect aroma profiles of obtained oils from changes.

It's needed more studies on the investigation of olive oils aroma profile for reveal the effects of harvesting time and method, olive varieties, climatic factors, storage conditions, and extraction techniques.

\section{References}

Aydar A., Öncü Öner, T., Ücok E.F. (2017), Effects of Hydroxytyrosol on Human Health. $11, \mathrm{pp} .147-157$.

Bayrak A., Hu H. (2013), Determination of Aroma Profiles of Olive Oils from Turkish Olive Cultivars, Journal of the American Oil Chemists Society, pp. 1281-1300. 
Boskou D., (2007), Olive oil, Chemistry and Technology, Second Ed., AOCS, ThessalonikiGreece.

Bouaziz M., Fki I., Jemai H., Ayadi M., Sayadi S. (2008), Effect of storage on refined and husk olive oils composition: Stabilization by addition of natural antioxidants from Chemlali olive leaves, Food Chemistry, 108(1), pp.253-262.

Cavalli J.F., Fernandez X., Cuvelier L.L., Loiseau A.M. (2004), Characterization of volatile compounds of French and Spanish virgin olive oils by HS - SPME of quality freshness markers Food Chemistry Characterization of volatile compounds of French and Spanish virgin olive oils by HS-SPME : Identification of quality-f, Food Chemistry, 88(1), pp. $151-157$.

Davis B.M. (2007), Volatile Organic Compounds and Antioxidants in Olive Oil: Their Analysis by Selected Ion Flow Tube Mass Spectrometry, University of Canterbury.

Fernandez M.D.L.A., SotoVargas V.C., Silva M.F. (2014), Phenolic Compounds and Antioxidant Capacity of Monovarietal Olive Oils Produced in Argentina, Journal of American Oil Chemists Society, 91, pp. 2021-2033.

Gomes da Silva M.D.R. et al. (2012), Olive Oil Composition : Volatile Compounds. in Dimitrios Boskou, (Ed.) Olive Oil - Constituents, Quality, Health Properties and Bioconversions, Intech Open Science, Shanghai, pp. 17-41.

Haddada F.M., Manai H., Daoud D., Fernandez X., Cuvelier L.L., Zarrouk M. (2007), Food Chemistry Profiles of volatile compounds from some monovarietal Tunisian virgin olive oils: Comparison with French PDO. Food Chemistry, 103(2), pp. 467-476.

Inarejos-García A.M., Gomez-Rico A., Salvador M.D., Fregapane G. (2009), Influence of malaxation conditions on virgin olive oil yield, overall quality and composition, European Food Research and Technology, 228, pp. 671-677.

Issaoui M., Hassine K.B., Flamini G., Brahmi F., Chehab H., Aouni Y., Mechri B., Zarrouk M., Hammami M. (2009), Discrimation of Some Tunisian Olive Oil Varieties Compounds and Chemometric Analysis. Journal of Food Lipids, 16, pp. 64-186.

Issaoui M., Flamini G., Brahmi F., Dabbou S., Ben Hassine K., Taamali A., Chehab H., Ellouz M., Zarrouk M., Hammami M. (2010), Effect of the growing area conditions on differentiation between Chemlali and Chétoui olive oils. Food Chemistry, 119 (1), pp. $220-225$.

Kaftan A. (2007), Farklı Yöre Zeytinlerinden Elde Edilen Naturel Zeytinyağının Duyusal Kalitesini Oluşturan Lezzet Maddelerinin SPME/GC/MS ve Lezzet Profili Analizi Teknikleri Kullanilarak Belirlenmesi. Ege University PhD thesis

Kesen S., Kelebek H., Sen K., Ulaş M., Selli S. (2013), GC - MS - olfactometric characterization of the key aroma compounds in Turkish olive oils by application of the aroma extract dilution analysis, Food Research International, 54(2), pp. 1987-1994.

Koprivnjak O., Conte L. (2002), Influence of Olive Fruit Storage in Bags on Oil Quality and Composition of Influence of Olive Fruit Storage in Bags on Oil Quality and Composition of Volatile Compounds, Food Technology and Biotechnology, 40(2), pp. 129-134.

Krichene D., Haddada F.M., Fernandez X., Cuvelier L.L., Zarrouk M. (2010), Original article Volatile compounds characterising Tunisian virgin olive oils : the influence of cultivar, International Journal of Food Science and Technology, 45, pp. 944-950.

Manai H., Haddada F.M., Oueslati I., Daoud D., Zarrouk M. (2008), Characterization of monovarietal virgin olive oils from six crossing varieties, Scientia Horticulturae, 115(3), pp. 252-260.

Morales M.T., Luna G., Aparicio R. (2005), Comparative study of virgin olive oil sensory defects, Food Chemistry, 91(2), pp. 293-301. 
Morales M.T., Aparicio R. (1999), Effect of extraction conditions on sensory quality of virgin olive oil, Journal of the American Oil Chemists' Society, 76, pp. 295-300.

Ozdemir Y., Tangu N.A., Ozturk A., Akçay M.E., Ercişli S. (2018), Fruit characteristics of six candidate olive cultivars, Folia Horticulturae, 30(1), pp. 169-177.

Pereira J.A., Casal S., Bento A., Oliveira M.B.P.P. (2002), Influence of Olive Storage Period on Oil Quality of Three Portuguese Cultivars of Olea europea, Cobrancosa, Madural, and Verdeal Transmontana, Journal of Agricultural and Food Chemistry, 50(22), pp. 6335-6340.

Rizzo V., Torri L., Muratore G. (2011), The Influence of Lighting Shelf Life of Extra Virgin Olive Oil Packaged in Coloured Pet Bottles, Italian Journal of Food Science, 33, pp. 30-34.

Servili M., Esposto S., Lodolini E., Selvaggini R., Taticchi A., Urbani S., Montedoro G., Serravalle M., Gucci R. (2007), Irrigation Effects on Quality, Phenolic Composition, and Selected Volatiles of Virgin Olive Oils Cv. Leccino, Journal of Agriculture and Food Chemistry, 55(16), pp. 6609-6618.

Servili M., Selvaggini R., Taticchi A., Esposto S., Montedoro G. (2003), Volatile Compounds and Phenolic Composition of Virgin Olive Oil: Optimization of Temperature and Time of Exposure of Olive Pastes to Air Contact during the Mechanical Extraction Process, Journal of Agriculture and Food Chemistry, 51(27), pp. 7980-7988.

Veillet S., Tomao V., Chemat F. (2010), Ultrasound assisted maceration: An original procedure for direct aromatisation of olive oil with basil, Food Chemistry, 123 (3), pp. 905-911.

Yang K.M., Cheng, M.C., Chen W.C., Tseng C.Y., Lin L.Y., Chiang P.Y. (2017), Characterization of Volatile Compounds with HS-SPME from Oxidized n-3 PUFA Rich Oils via Rancimat Tests, Journal of Oleo Science, 66(2), pp. 113-122. 